Fetal Diagnosis and Therapy

\title{
The Diagnostic Features of Spina Bifida: The Role of Ultrasound
}

\author{
Beverly G. Coleman Jill E. Langer Steven C. Horii \\ Division of Abdominal Imaging, Radiology Department, Hospital of the University of Pennsylvania, Philadelphia, Pa., USA
}

\section{Key Words}

Fetal ultrasound - Spina bifida - Myelomeningocele .

Ventriculomegaly · Hindbrain herniation - Syrinx - Kyphosis . Scoliosis

\begin{abstract}
Myelomeningocele (MMC) is one of the most devastating, nonlethal congenital anomalies worldwide. The live birth prevalence of MMC changed dramatically in the 1980s with the introduction of maternal serum screening and the widespread use of prenatal ultrasound imaging. The high-resolution ultrasound affordable today with state-of-the-art equipment allows us to make a very accurate diagnosis of MMC, including details related to the entire fetal central nervous system. Ultrasound can accurately localize the site of the osseous and soft tissue defects. Congenital spinal defects can be characterized definitively as open or closed, which are treated very differently with in utero repair, which is done in some cases, compared to only conservative follow-up with postnatal therapy for occult defects. Additional findings of kyphosis, scoliosis and anomalous vertebrate and associated conditions such as cervical syrinx can be identified. The state of the intracranial structures, including the presence or absence of ventriculomegaly and hindbrain herniation, as well
\end{abstract}

as unexpected complications such as intracranial hemorrhage can be diagnosed. The severity of neurological compromise in some fetuses can be estimated by detailed examination of the lower extremities. As well as searching for talipes, we also now routinely characterize flexion and extension motions at the hip, knee and ankle joints. The information provided by ultrasound plays a crucial role, now more than ever, in patient counseling and pregnancy management. This article emphasizes how we utilize ultrasound in the evaluation of patients with suspected MMC at the Center for Fetal Diagnosis and Treatment at the Children's Hospital of Philadelphia.

(c) 2014 S. Karger AG, Basel

\section{How We Do It: Protocol and Technique}

It is essential that every second-trimester fetal anatomic survey includes a complete evaluation of the spine. Images should be recorded in longitudinal, sagittal, coronal and axial planes. The axial view demonstrates three ossification centers, a centrum for the vertebral body and two lateral masses that converge, creating a triangular configuration (fig. 1a). The sagittal view demonstrates the spine as two parallel echogenic lines representing the cen-

\section{KARGER 125}

(c) 2014 S. Karger AG, Base

$1015-3837 / 14 / 0373-0179 \$ 39.50 / 0$

E-Mail karger@karger.com

www.karger.com/fdt
Beverly G. Coleman, MD, FACR

Professor of Radiology Associate Chair, Radiology Department

Division of Abdominal Imaging, Hospital of the University of Pennsylvania

3400 Spruce Street, Philadelphia, PA 19104 (USA)

E-Mail beverly.coleman@uphs.upenn.edu 
Fig. 1. Normal spine with a 9-MHz transducer at 20 weeks and 3 days. a Axial image of the spine with 2 lateral masses (arrows) that converge and a centrum for the vertebral body (curved arrow). b Sagittal image of 2 parallel echogenic lines (arrows) that taper distally in the sacral region.
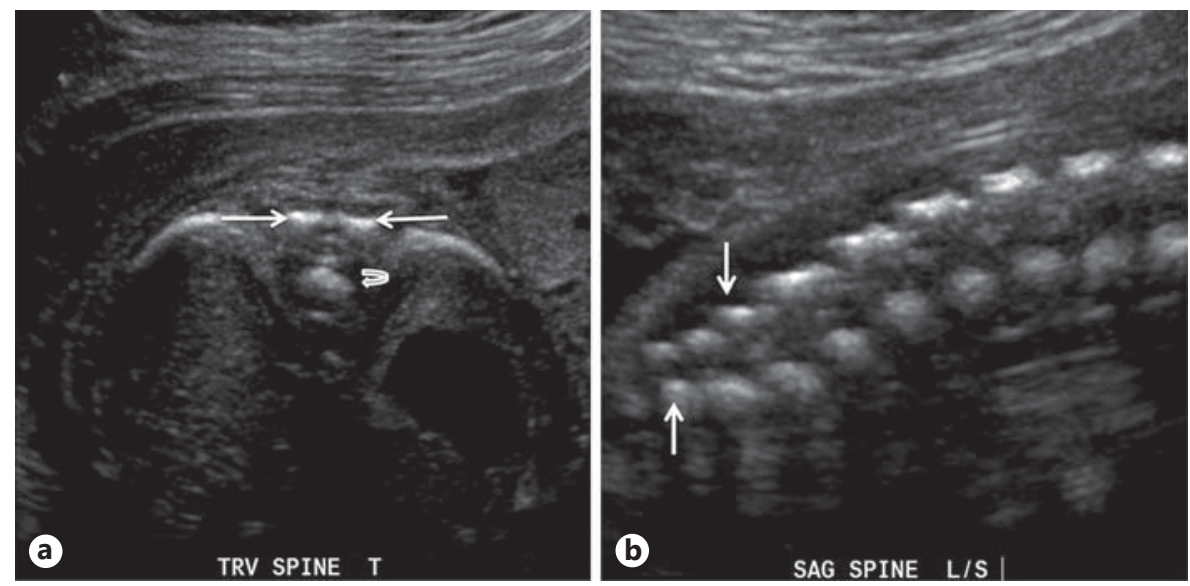

Fig. 2. Localization of the conus medullaris with a $12-\mathrm{MHz}$ transducer at 29 weeks and 2 days. a Sagittal image of the spine with the arrow-shaped tip of the conus medullaris (arrow). b Sagittal image of the spine with a low conus medullaris (arrow) at the bottom of S4 at 25 weeks.
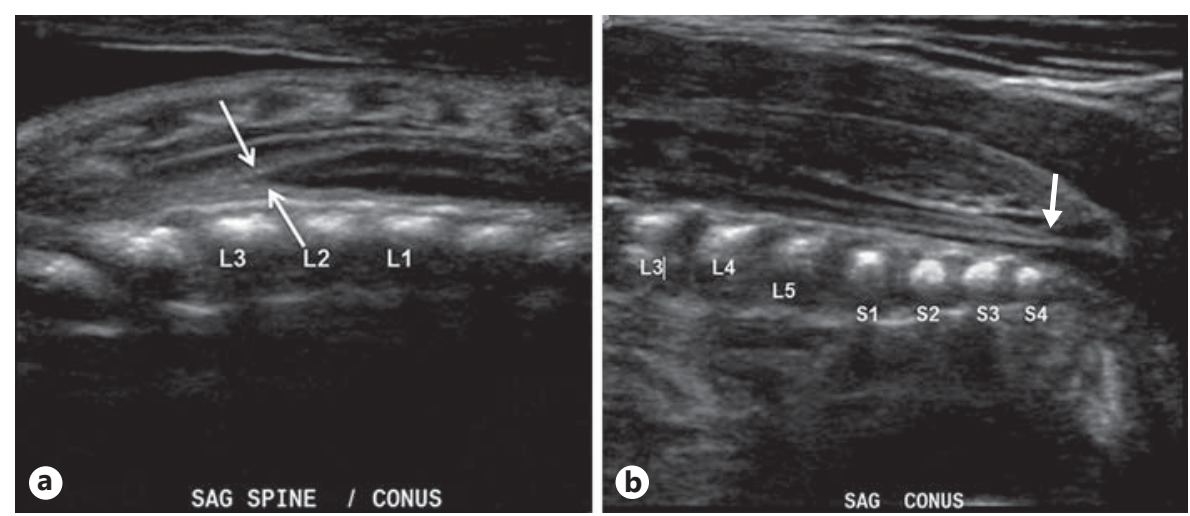

trum and lamina with a subtle normal lordosis at the cervical and lumbar levels and a subtle kyphosis at the thoracic level, with convergence of these lines distally (fig. 1b). In the coronal plane, the posterior elements appear as paired, echogenic lines with subtle flaring at the lumbar level and tapering at the sacral level.

For the detailed examinations we perform at the Center for Fetal Diagnosis and Treatment (CFDT) at the Children's Hospital of Philadelphia, we use more than one transducer, with frequencies ranging from 5 to 12 $\mathrm{MHz}$. We also use various techniques to 'position' the fetus because it is optimal to examine the spine when it is in the near field as well as to evaluate the spine away from the uterine wall. When examining the spine in the axial plane, the scan angle is also a very important factor. Maternal body habitus and incomplete ossification of the fetal spine are two factors that can negatively impact scan quality. When the transabdominal (TA) scan is not optimal, transvaginal (TV) scans are done to evaluate the spine, especially when the fetus is in a frank breech position and, similarly, to image the head when the fetus is in a low cephalic position. Multiple images including cine clips and 3D scans are routinely recorded. If the initial scans are performed in the late first or early second trimester, vertebral ossification is often incomplete, so follow-up scans may be needed. It is generally accepted that when using only the TA approach, there is adequate ossification to L 5 by 16 weeks and to S2 by 22 weeks [1]. Some authors have reported neural arch ossification by 13 weeks, and successful evaluation of the spinal soft tissues and osseous structures can be performed using TA and TV imaging [2].

Our spine examination includes localization of the conus medullaris using the highest-frequency transducer possible (fig. 2). This position varies with gestational age, typically with the conus medullaris ending at L4 or higher by 13-18 weeks, L3 or higher by 19-36 weeks and L2 or higher after 36 weeks [3]. The most caudal vertebrae with a corresponding rib represent the T12 level in the vast majority of cases. In the mid-second trimester, the lowest and most faintly ossified vertebrae represent S4. In counting down from T12 or up from S4, it is possible to determine the conus medullaris level and accurately localize spinal defects. The osseous defect is evident as a subtle diver- 

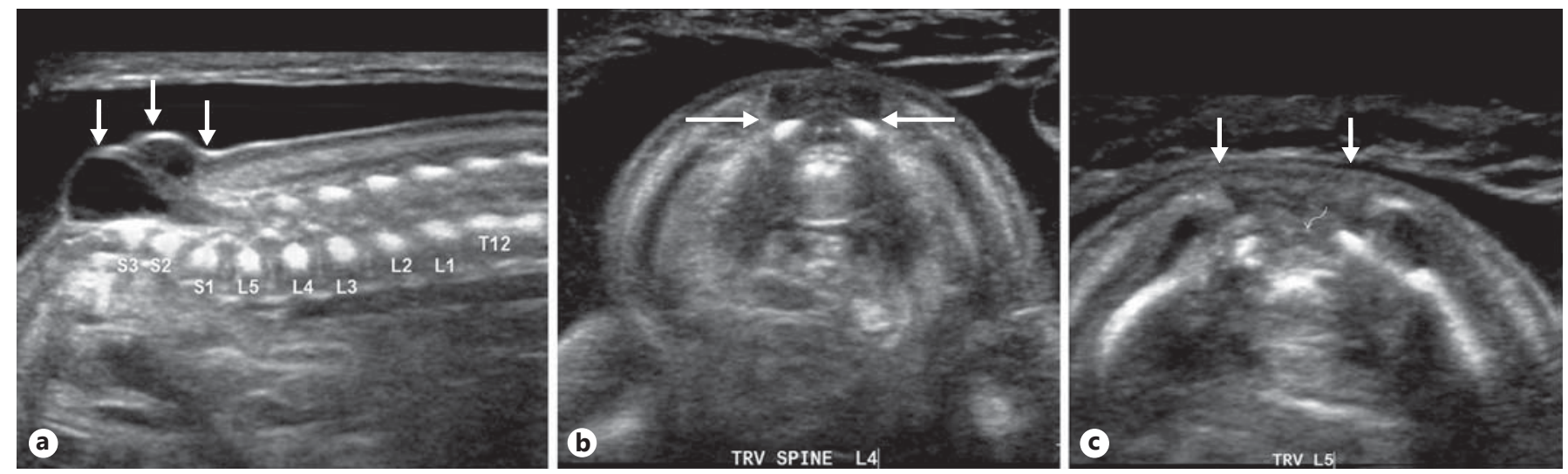

Fig. 3. MMC level determination from L4 to S4 at 19 weeks and 1 day. a Sagittal image of a small sac (arrows) with neural elements. The skin and subcutaneous tissues are deficient at the top of S2. b Axial image of divergence of the posterior arches at L4 (arrows) at the site of the osseous defect. c Axial image of increased divergence of the ossification centers and intact skin (arrows) at L5 with tethered cord (curved arrow).
Fig. 4. Estimation of sac size at 19 weeks and 4 days. a Sagittal image of MMC sac (arrow) with cursors placed for anteroposterior diameter and length. b Axial image of the sac width, $1.5 \mathrm{~cm}$ (cursors), with neural elements (arrows).
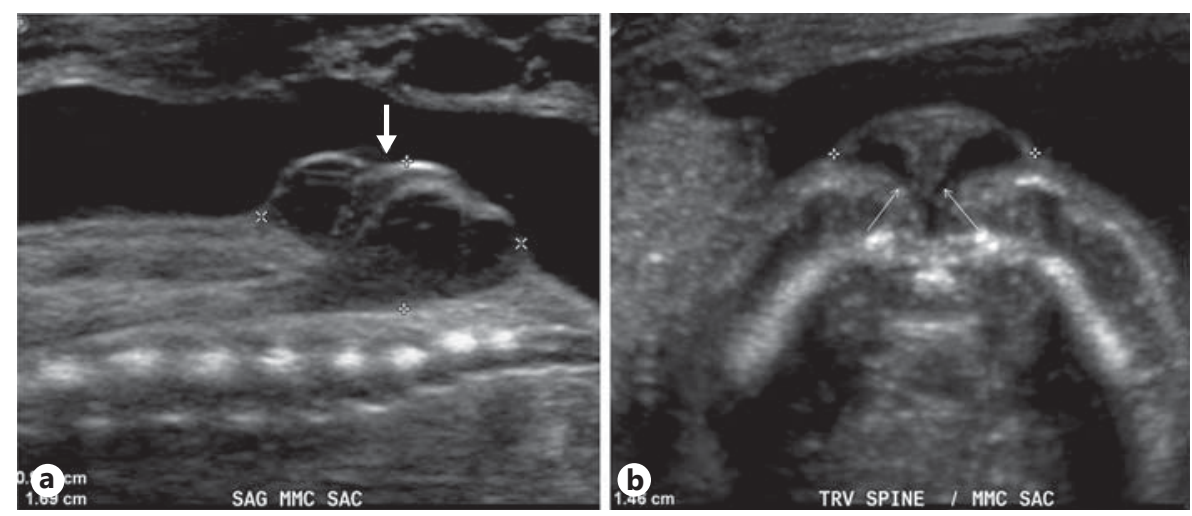

gence of the lateral masses into a $\mathrm{V}$ shape. This typically occurs at one or more levels above the skin defect where the subcutaneous tissues and muscles are deficient (fig. 3). At the level of the osseous defect, all of the superficial soft tissues and muscles are generally intact.

It has been previously reported that fetal magnetic resonance imaging (MRI) and ultrasound are equally effective in determining the lesion level in the fetus with myelomeningocele (MMC) [4]. However, our experience does not support this conclusion. At the CFDT, ultrasound was relied upon for lesion level determination in all referred fetal MMC cases for the Management of Myelomeningocele Study (MOMS). On the other hand, MRI was deemed definitive for the presence or absence of hindbrain herniation, which was crucial in the selection of candidates for in utero surgical repair [5]. We have maintained this methodology in our tertiary center since the trial was stopped for efficacy in December 2010.

Diagnostic Features of Spina Bifida: Role of Ultrasound
In addition to localization of the conus medullaris and spinal defects, we routinely measure any visualized sac in three dimensions, anterior-posterior diameter and length on the longitudinal view as well as width on the axial view (fig. 4). The MMC size determination is important for the planning of the fetal surgical repair because a regenerative tissue matrix may be required for the skin closure of very large MMC defects. The components of any sac are assessed to determine the presence of neural elements which appear as linear, echogenic areas surrounded by anechoic cerebrospinal fluid (CSF). The neural placode in an MMC represents the exposed spinal cord at the skin surface which appears as a hypoechoic, ovoid region within the sac (fig. 5). The wall of an MMC is assessed for thickness and characterized as thin or thick and, if thick, whether it is completely or partially skin covered based on a symmetric or asymmetric wall appearance (fig. 6). The entire spine is also evaluated for any anomalous ver- 
Fig. 5. MMC with exposed spinal cord at 21 weeks and 4 days. a Coronal image of neural placode (arrows) within a $2.2-\mathrm{cm}$ sac. b Axial image of osseous defect at L2 with tethered cord (arrow) toward the right with surrounding CSF (curved arrow). The skin and muscle are intact. c Axial image of skin defect at L4 (arrow) with anomalous ossification center for the vertebral body (curved arrows) at L4. d Sagittal image of large sac from L4 to S2 with reconstitution of the skin and soft tissues at S3-4 (arrows).
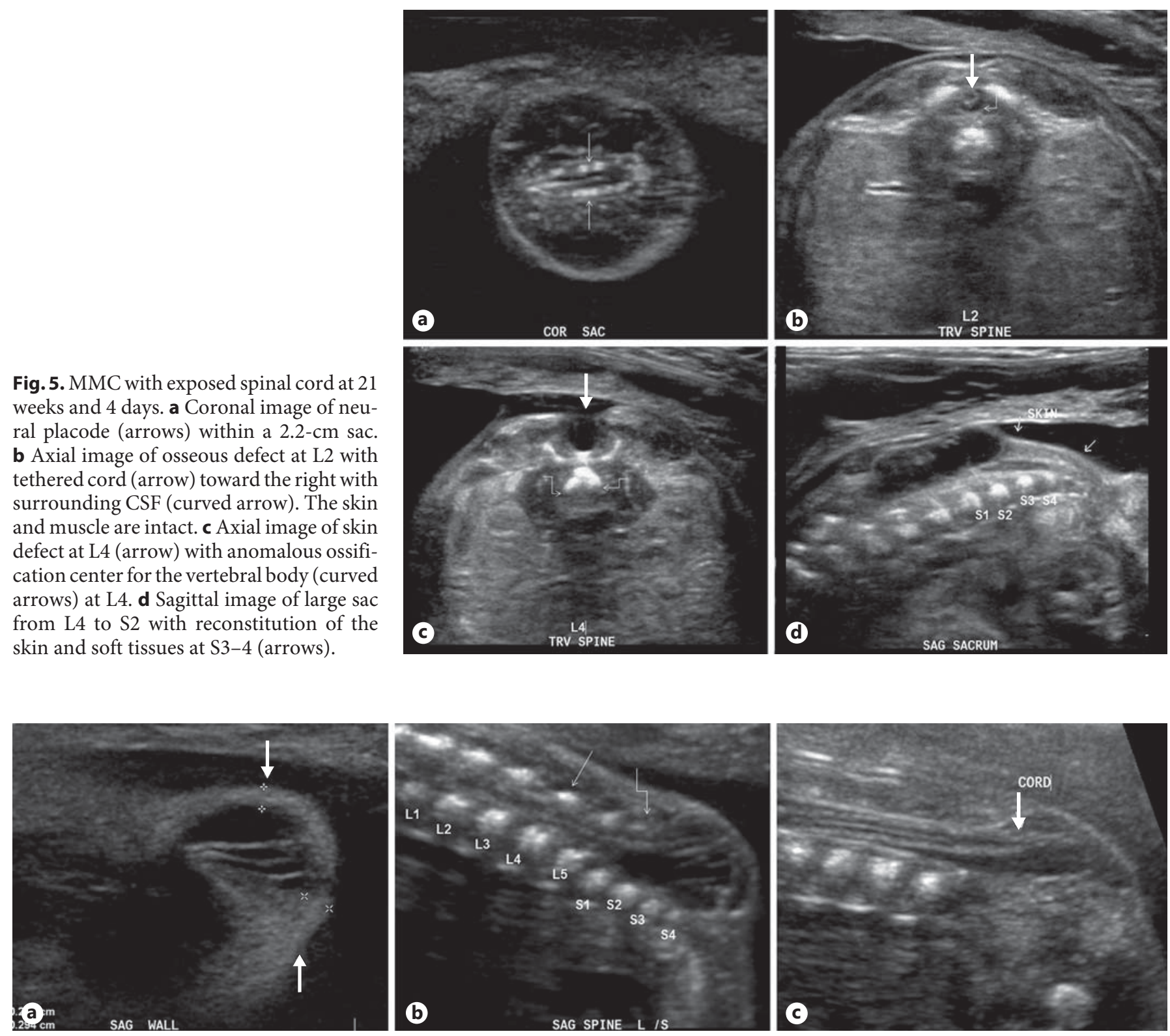

Fig. 6. Measurement of sac wall at 22 weeks and 1 day. a Coronal image of a small, skin-covered sac (arrows) with a thickened wall measuring over $2 \mathrm{~mm}$. b Sagittal image of osseous defect at L4 (ar- row) and skin defect at S2 (curved arrow). c Sagittal image of the conus medullaris (arrow) extending into the small sac, indicating the presence of neural elements and a tethered cord. tebrae and the presence of scoliosis, kyphosis or other abnormalities.

Our protocol includes axial, sagittal and coronal images of the cerebral ventricles, with both lateral ventricles and the third ventricle routinely measured on axial images. The ependymal lining of the ventricles, choroid plexus and germinal matrix are subjectively evaluated for nodularity, increased echogenicity and any signs of intra- ventricular hemorrhage. The cerebellum and cisterna magna are scanned in two planes, axial and coronal, to assess for hindbrain herniation. The lower extremities are subjectively evaluated for spontaneous motion from the hip to the toes, for any foot deformities (most often talipes and occasionally rocker bottom foot) and for foreleg muscle development, with flexion and extension at all joints recorded (fig. 7). 
Fig. 7. Bilateral talipes of variable severity. a 3D image of talipes at 21 weeks and 5 days, with spontaneous lower extremity motion observed on real-time images. $\mathbf{b} 3 \mathrm{D}$ image of talipes at 23 weeks with the lower extremities persistently crossed at the ankles, with limited motion. c 2D image of severe talipes with atrophy of the muscle and soft tissues (arrows) above the ankle joint at 21 weeks and 5 days. $\mathbf{d}$ 3D image of fixed flexion deformity at the ankle (arrow) and extension at the knee joint (arrowhead) at 22 weeks.
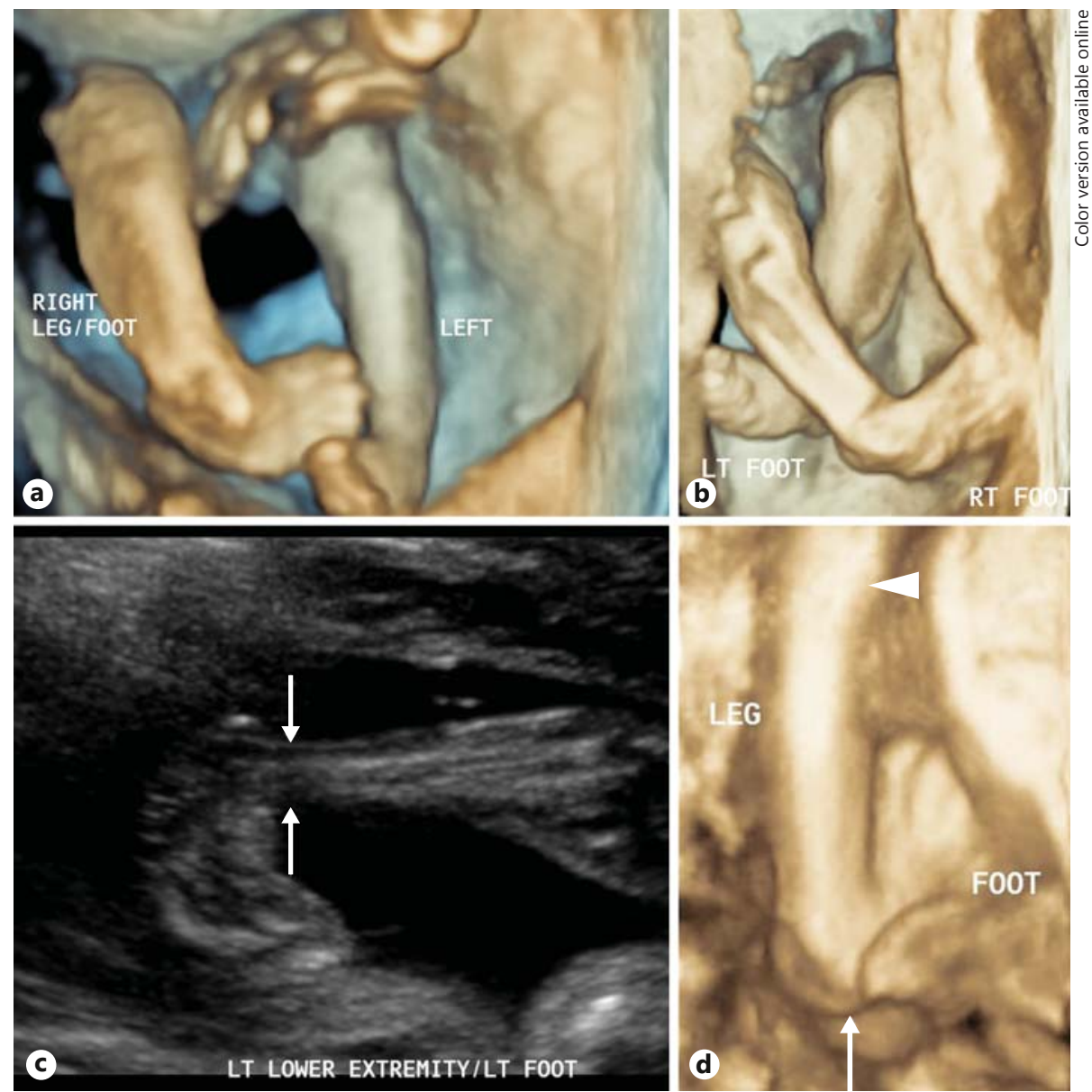

\section{Classification of Spinal Defects}

Neural tube defects can be classified as spina bifida (SB) occulta, 'closed' with no exposure of the neural tissue to amniotic fluid, or SB aperta, 'open' SB. Imaging is important because laboratory tests, such as maternal serum and amniotic fluid $\alpha$-fetoprotein and amniotic fluid acetylcholinesterase, are normal in occult defects. In addition, there are no secondary cranial findings in occult defects to serve as a tip-off of spinal dysraphism. The prenatal diagnosis in such cases is more challenging, thus optimal visualization of every spinal segment is required.

\section{Occult Spinal Defects}

The reported prevalence of occult spinal defects varies widely depending largely on the age of the patients included in a specific study. The most accurate estimate of the occurrence rate is $17 \%$ of examined spines [6]. There is a significant association with cutaneous markers such as lipomas, dermal sinus tracts, hemangiomas and, most notably, hypertrichosis [6, 7]. Fetuses with occult spinal defects are routinely managed postnatally as the spinal nerves and cord are normal. The various types of occult spinal dysraphism include tethered cord, diastematomyelia, subcutaneous or intraspinal lipoma, thickened filum terminale, dermoid or epidermoid cyst, and myelocystocele (fig. 8).

\section{Tethered Cord}

A tethered spinal cord can be diagnosed if the conus medullaris lies inferior to L3-4 after 18 weeks (fig. 2). Open or closed defects are associated with a tethered cord in up to $100 \%$ of cases (fig. 3, 6, 7). The tethered cord on axial scans appears as the 'central dot' signature closely applied to the lamina in the nondependent posterior portion of the canal, with CSF between the ventral wall of the canal and the anterior aspect of the cord (fig. 5). On sagittal scans, the conus medullaris typically extends far below L3 and often to the exact site of the defect in SB. 

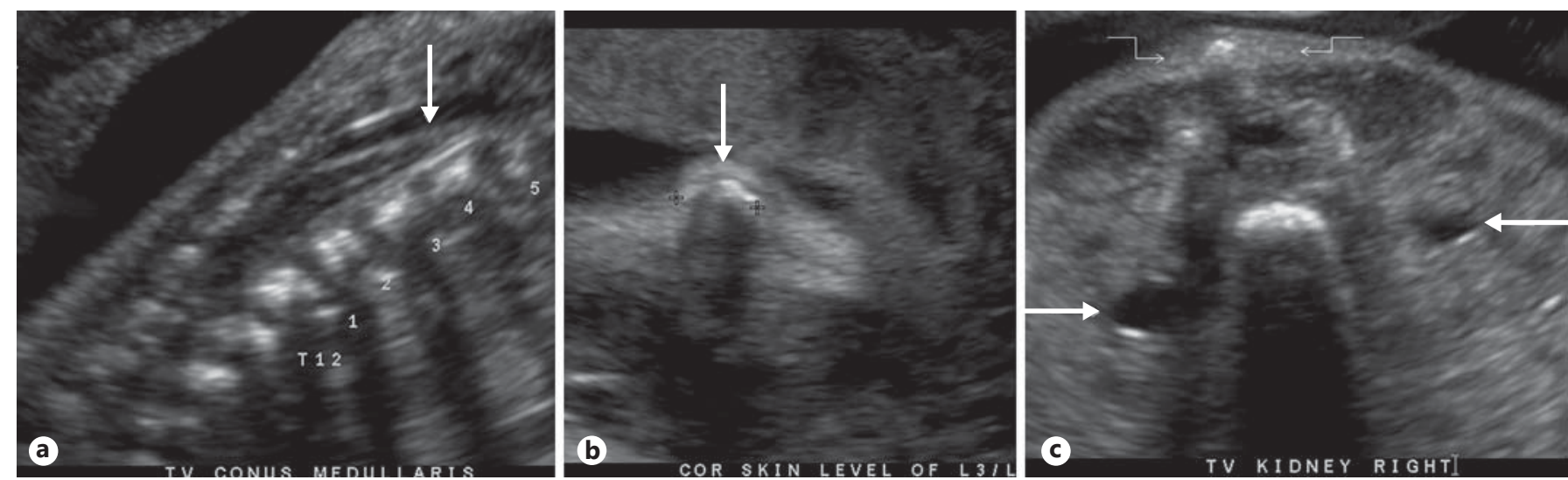

Fig. 8. Occult spinal dysraphism in a frank breech fetus at 23 weeks and 4 days. a Coronal TV image of a tethered cord with conus medullaris (arrow) at the bottom of L4. b Coronal image of an echogenic, shadowing focus (arrow) within the skin, measuring just over $1 \mathrm{~cm}$ at the level of L3-4. c Axial image of the echogenic focus in thickened skin (curved arrows) external to the muscles. Mild renal pyelectasis (arrows).
Fig. 9. Diastematomyelia involving the caudal thoracic spinal cord from T7 to T11 at 25 weeks and 3 days. a Axial image of the untethered cord (arrow) at T12 with the central dot signature. $\mathbf{b}$ Axial image of the hemicords (arrows) converging at T11 with an anomalous left posterior arch (curved arrow). c Axial image of 2 distinct hemicords (arrows). The ribs were also anomalous and asymmetric (curved arrows). d Sagittal image of the hemicords (arrows) with anomalous thoracic vertebrae (curved arrow).
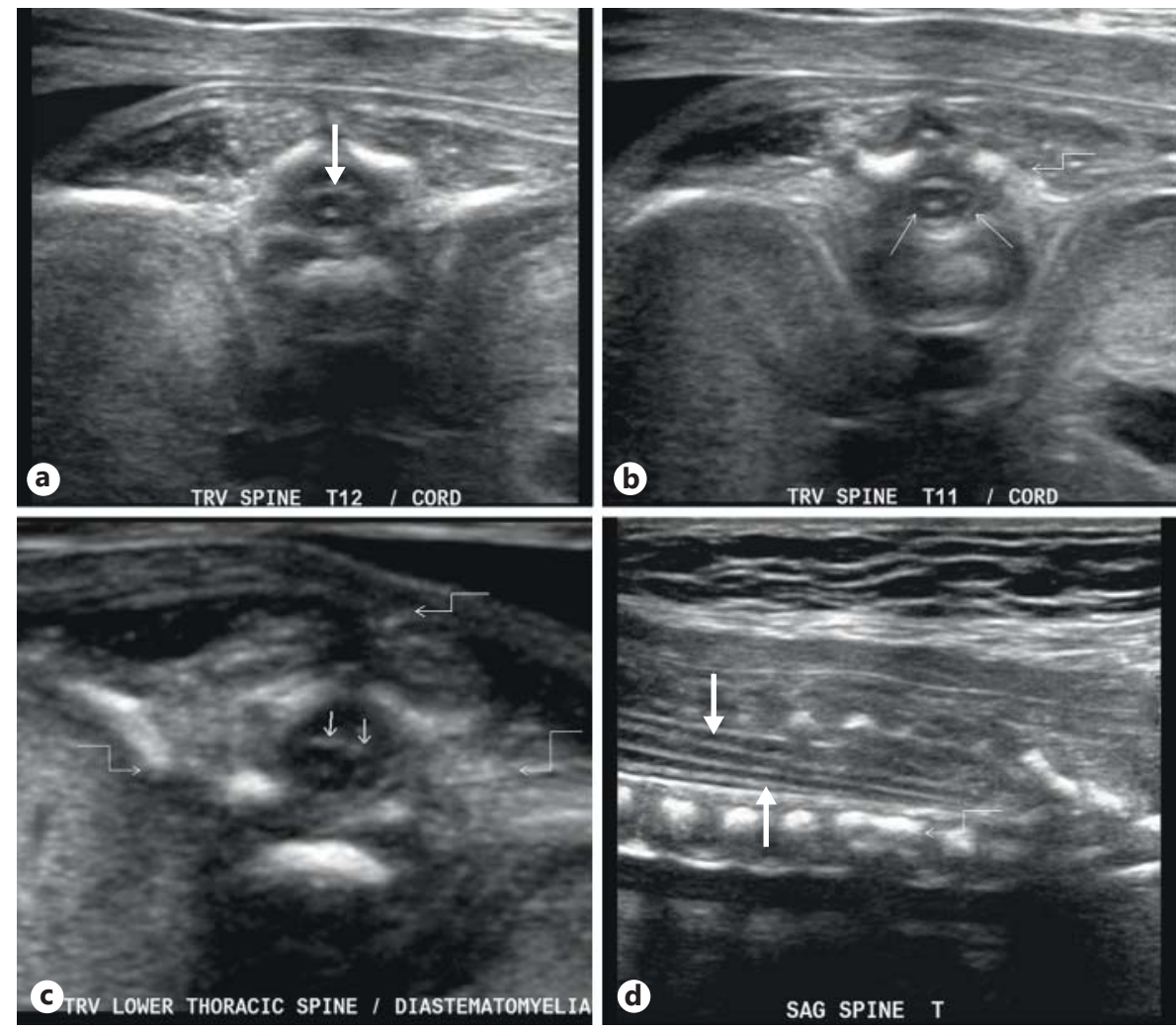

\section{Diastematomyelia}

Diastematomyelia or split cord malformation is a complete or incomplete division of the spinal cord into two hemicords with a single or separate dural sac and arachnoid space (fig. 9). There is a sagittal septum - osseous, cartilaginous or fibrous - in the spinal canal that may be associated with hydromyelia, segmental anomalous vertebrae or visceral abnormalities such as genitourinary or anorectal malformations (fig. 10). The sagittal cleft may involve the spinal cord, conus medullaris or filum terminale. When there are no other associated spinal anomalies, the prognosis is favorable, with normal a-fetoprotein and amniotic fluid acetylcholinesterase levels [8]. 
Fig. 10. Diastematomyelia with osseous septum at 18 weeks and 3 days. a Axial image of osseous septum (arrow) splitting the cord at L3. b Sagittal image of osseous septum (arrow) within the central canal with the conus at L5 (curved arrow).

Fig. 11. Lipomyelomeningocele referred for suspected sacrococcygeal teratoma at 24 weeks and 1 day. a Sagittal image of thick walled sac (arrows) with echogenic foci in the sac and subcutaneous tissues (curved arrows). b Axial images of solid focus (cursors) with adjacent, thickened skin (arrows).

Fig. 12. Lipomyelomeningocele referred at 21 weeks and 4 days for sacral agenesis. a Sagittal image of focal lipoma (arrow) as echogenic mass in the superficial tissues. b Coronal image of sac with thick highly echogenic wall (arrow) and neural canal echogenic tissue (curved arrow).
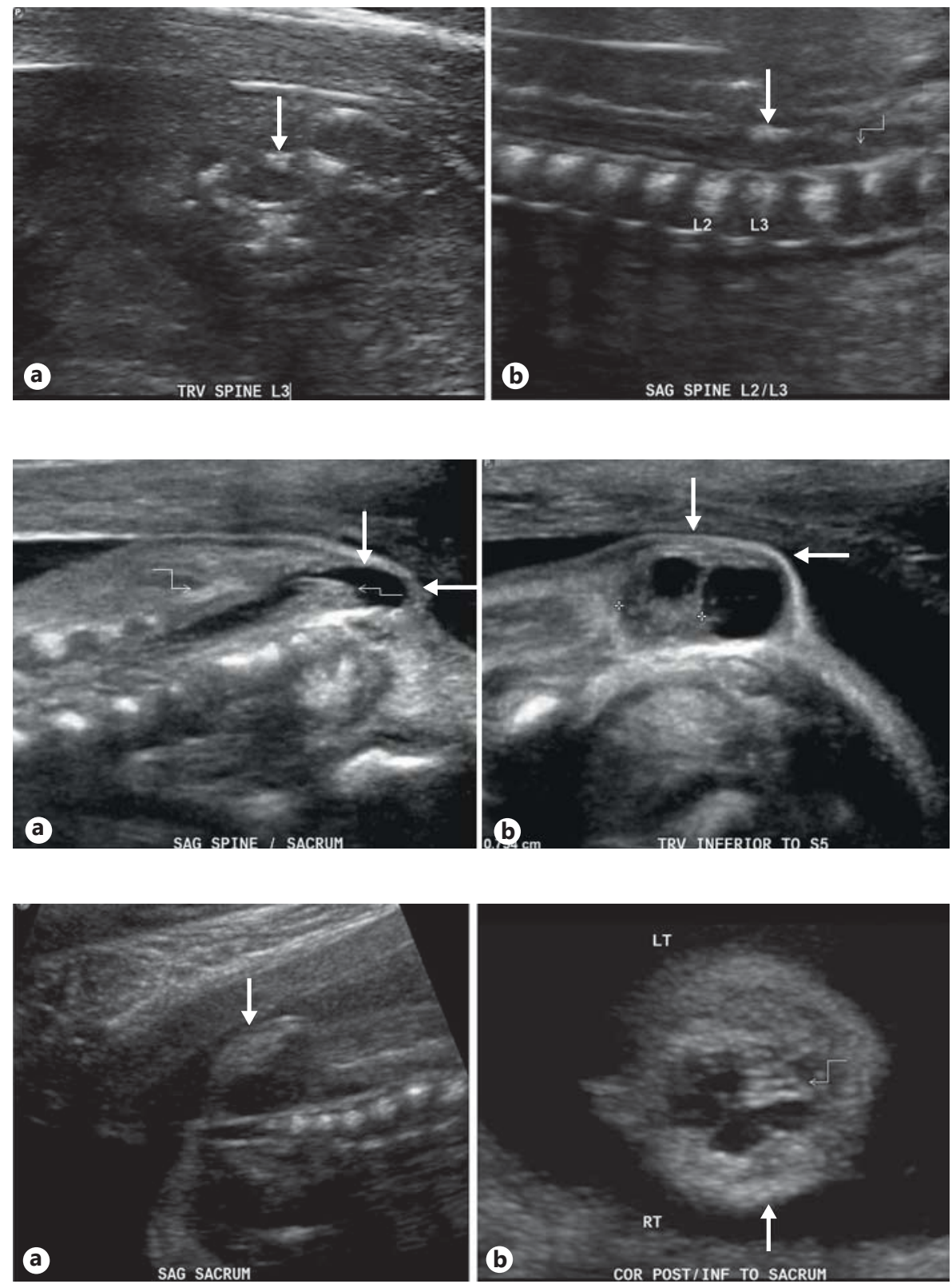

\section{Lipomyelomeningocele}

Lipomyelomeningocele is a complex disorder within the spectrum of closed neural tube defects. A subcutaneous lipoma, generally in the lumbosacral region, extends through a defect in the fascia and vertebral neural arch, tethering the cord to the adjacent dura and soft tissues (fig. 11). This defect may also be associated with other conditions such as diastematomyelia, hydromyelia and scoliosis [9].

The higher resolution afforded by state-of-the-art scanners coupled with detailed spinal imaging has im- proved our ability to detect a lipoma as a discrete, very echogenic mass (fig. 12). MRI has also proven to be very useful in substantiating the diagnosis, using imaging sequences that can delineate the highly intense fat-containing tissues distinct from the overlying skin at the base of a thick walled sac. Associated anomalies, including sacral agenesis in $20 \%$ and anorectal and genitourinary tract malformations in $5-10 \%$, can often be diagnosed with confidence on both prenatal ultrasound and MRI [10]. 
Fig. 13. Terminal myelocystocele at 22 weeks. a Sagittal image of cystic dilatation of the central canal (arrows) extending into a small sacral sac with low conus medullaris. b Coronal image of the sac with a thickened wall (cursors), internal cyst (arrows) and no neural elements.
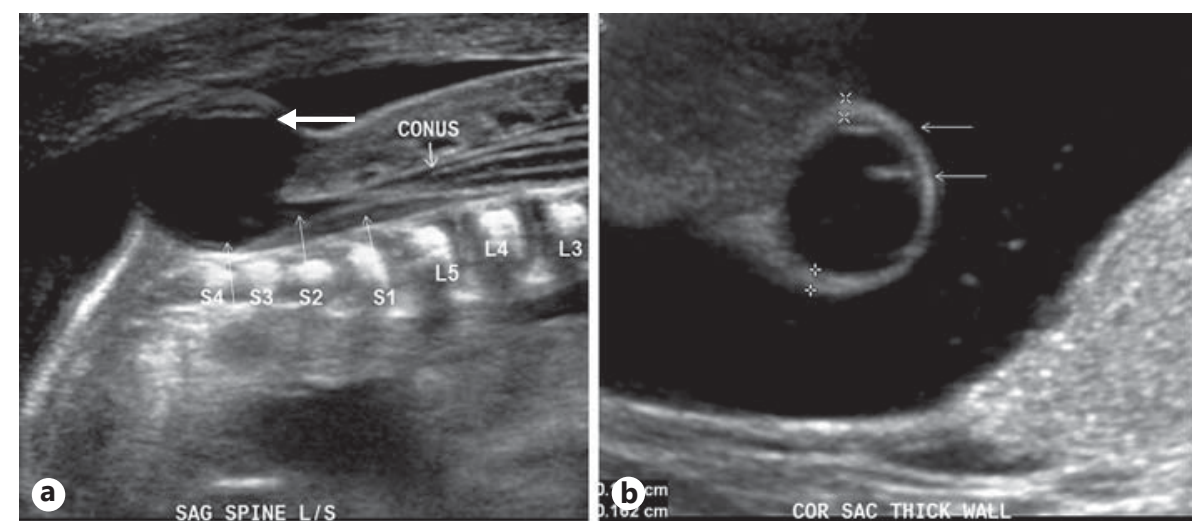

Fig. 14. Terminal myelocystocele at 29
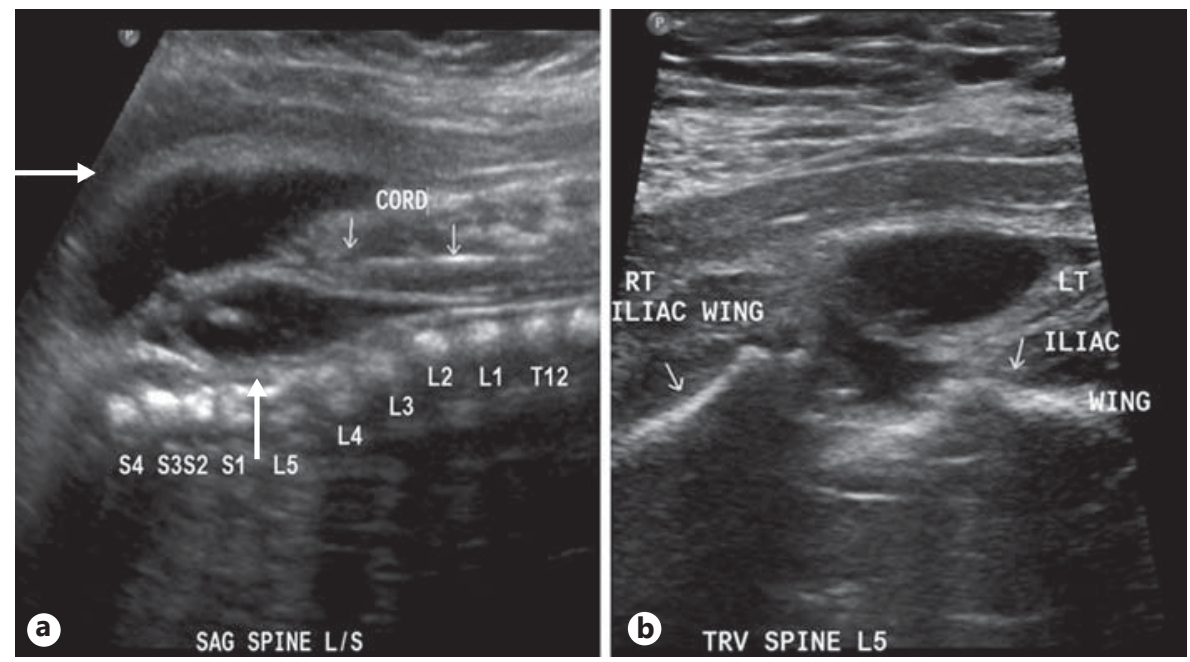
weeks. a Sagittal image of a large sac (ar rows) with a 'cyst within a cyst' appearance. b Axial image at L5 with abnormal iliac bones which are widely splayed (arrows).

Terminal Myelocystocele

Terminal myelocystocele is a rare type of occult spinal dysraphism constituting approximately $3.5-7 \%$ of skincovered lower spinal defects [11]. This appears as an ependymal lined sac that corresponds to a cystic dilatation of the central canal with overlying arachnoid and CSF that herniates via a defect in the posterior arches of the lumbosacral spine (fig. 13). When the myelocystocele is isolated without other associated midline defects, the outcome is generally more favorable than for patients with an atypical MMC. A meningocele collects around the main terminal cyst, so the mass may have a double wall with a 'cyst within cyst appearance' (fig. 14). The mass is skin covered, typically with a large, thick-walled sac that may significantly increase in size when monitored serially over time [12].

The terminal myelocystocele may be associated with various anomalies that include malformations of the abdominal wall, intestinal tract, genitourinary system, pel- vis and lower spine [13]. Nonterminal myelocystoceles are located in the cervical, thoracic or upper lumbar spine (fig. 15) and are much rarer than terminal myelocystoceles [14].

\section{Open Spinal Defects}

MMC, Meningocele and Myeloschisis

The vast majority of spinal defects are classified as open (fig. 3-6). Prenatal diagnosis in low-risk patients is generally made at the time of routine scanning for the fetal anatomic survey. However, diagnosis with ultrasound has been reported as early as the first trimester. Regardless of gestational age, meticulous scanning is always required in these cases [15]. The accuracy of tertiary centers such as ours is virtually $100 \%$ and is impacted by the experience of the sonographer and imaging physician, the resolution of the ultrasound equipment and the amount of time dedicated to the scan [16]. 
Fig. 15. Cervical myelocystocele at 18 weeks and 3 days. a Sagittal image of a small, thick-walled sac (arrow) in the posterior nuchal region (cursors). b Axial image at the level of $\mathrm{C} 2$ with subtle soft tissue defect (arrows) and divergence of the posterior arches.

Fig. 16. Skin-covered meningocele at 30 weeks and 2 days. a Axial image of small, asymmetrically thickened sac (arrow) at L4. b Sagittal images of a thin tract (arrows) to the meningocele sac with conus medullaris within the canal (curved arrow).

Fig. 17. Lumbosacral myeloschisis at 21 weeks and 2 days. a Sagittal image with osseous defect at L3 extending to S3 (arrows), with no sac and tethered cord. b Axial image of widely splayed posterior arches at L5 (arrows).
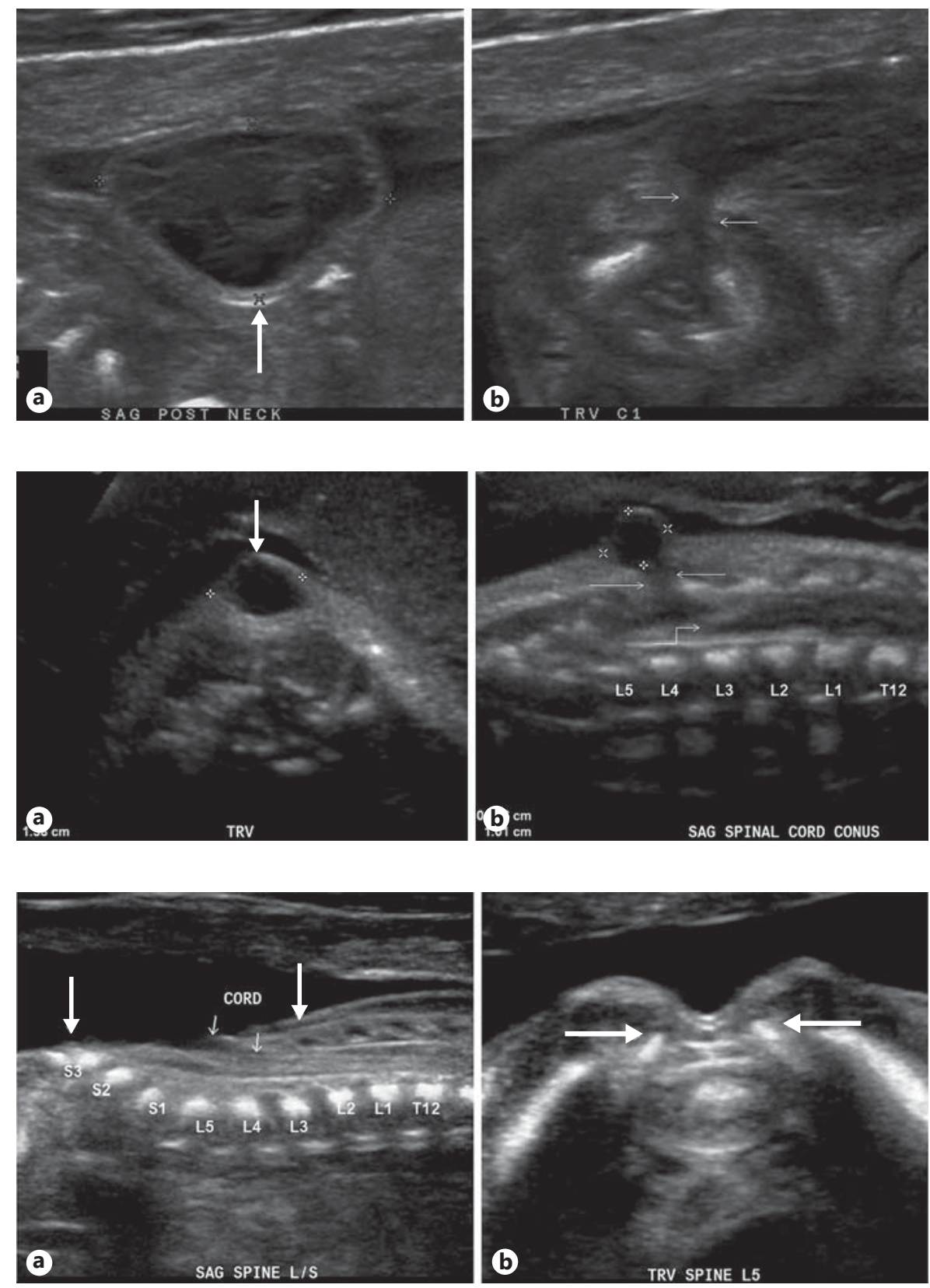

High-resolution ultrasound equipment has facilitated earlier detection and more accurate characterization of MMC and other types of spinal dysraphism. At the CFDT, we examine such defects daily, with MMC being the most common spinal dysraphism we have encountered before, during and after the MOMS trial. The most frequent type of open spinal defect is MMC, which appears on sonography as a complex lesion containing neural elements, the actual neural placode (fig. 5), nerve roots, meninges and
CSF. A meningocele has protrusion of only the meninges, forming a purely cystic lesion that contains CSF with a sac wall that is composed typically of a very thin arachnoid membrane. The spinal cord and nerve roots are located within the canal and tend to be relatively normal (fig. 16). In approximately $20 \%$ of open defects, there is a flattened cord with or without a complete or partial thin membrane but without a well-defined, measurable sac representing a myeloschisis defect [16] (fig. 17, 18). 
Fig. 18. Lumbosacral myeloschisis at 20 weeks and 1 day. a Sagittal image of a flat defect with an incomplete, very thin membrane (arrow) in the most distal aspect but no measurable sac. b Axial image of the skin defect (arrow) and the anomalous ossification center for the vertebral body (curved arrow) at L4.
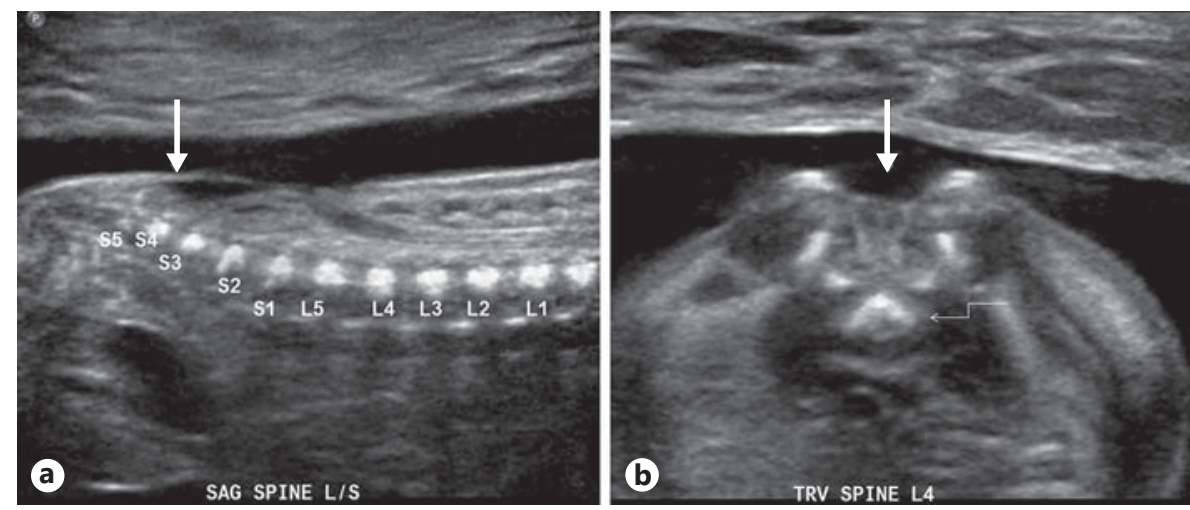

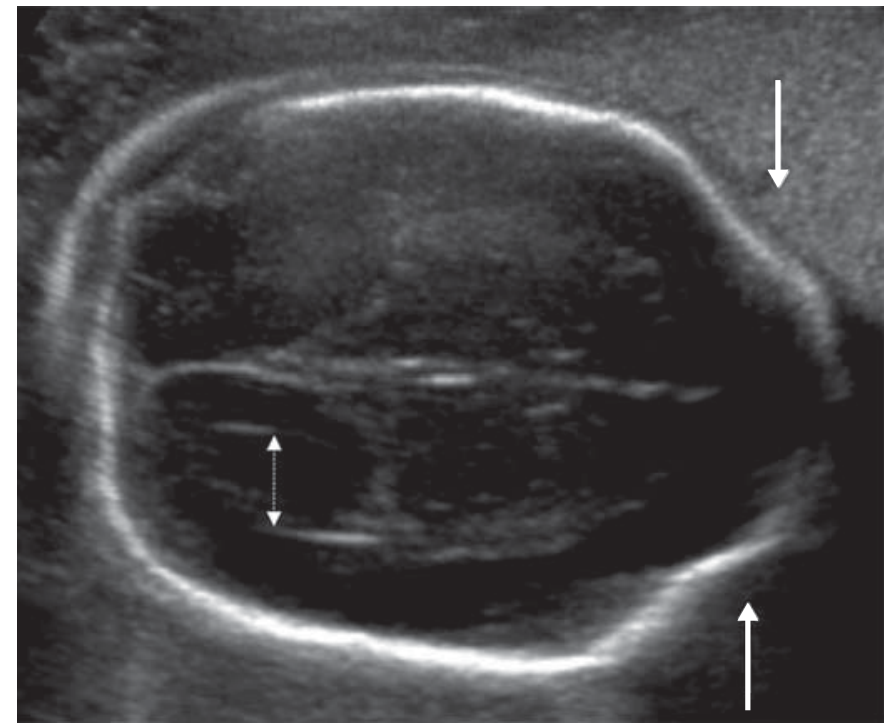

Fig. 19. Lemon sign at 22 weeks and 6 days. Axial image of the head of the fetus with a lumbosacral MMC demonstrates frontal concavity of the skull (arrows), producing the lemon sign and mild ventriculomegaly (dashed arrows).

\section{Cranial Findings in Spinal Dysraphism}

\section{Frontal Bone Concavity: 'Lemon Sign'}

Prenatal detection of MMC by fetal ultrasound was greatly improved in the mid-1980s by the description of a number of characteristic cranial findings which were found to be more easily appreciated than directly visualizing the spinal defect [17-19]. The 'lemon sign' describes the shape of the skull in the transverse plane present in many fetuses with MMC and is typified by concavity of the frontal bones near the coronal sutures as opposed to the convex configuration of the normal fetal cranium [17-19] (fig. 19). It is likely that the etiology of the concavity is re-

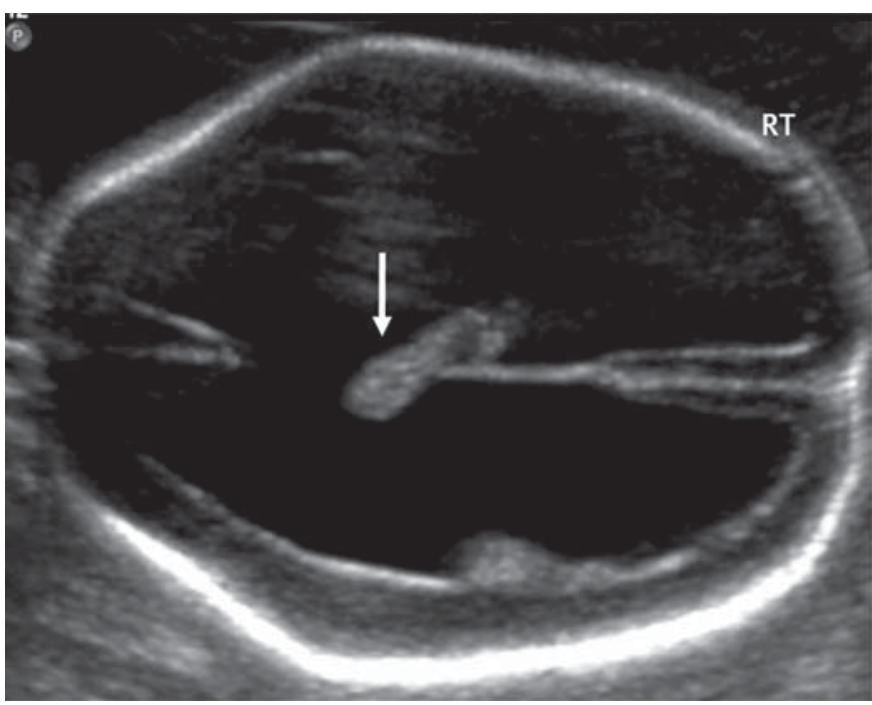

Fig. 20. Ventriculomegaly at 20 weeks and 3 days. Axial image of the head of a fetus with a lumbosacral myeloschisis demonstrates ventriculomegaly with both lateral cerebral ventricles dilated to 13 $\mathrm{mm}$ as well as frontal concavity. The nondependent choroid plexus (arrow) is noted to have prolapsed through an opening in the fenestrated intraventricular septum into the left ventricle.

lated to low hydrostatic pressure due to leakage of CSF from the open neural tube defect into the amniotic fluid [20]. It has been theorized that the developing frontal bones may be the most susceptible part of the cranium to this effect, resulting in the abnormal shape [20,21]. The lemon sign has been described in affected fetuses with MMC as early as 13 weeks, in $50-90 \%$ before 24 weeks, but only in $13 \%$ after 24 weeks [21-23]. In cases monitored with serial sonographic examinations, the frontal concavity has been observed to resolve after the second trimester, presumably due to further ossification and strengthening of the calvarium later in gestation [21,24]. It is important to note that the lemon sign is not specific or diagnostic for 

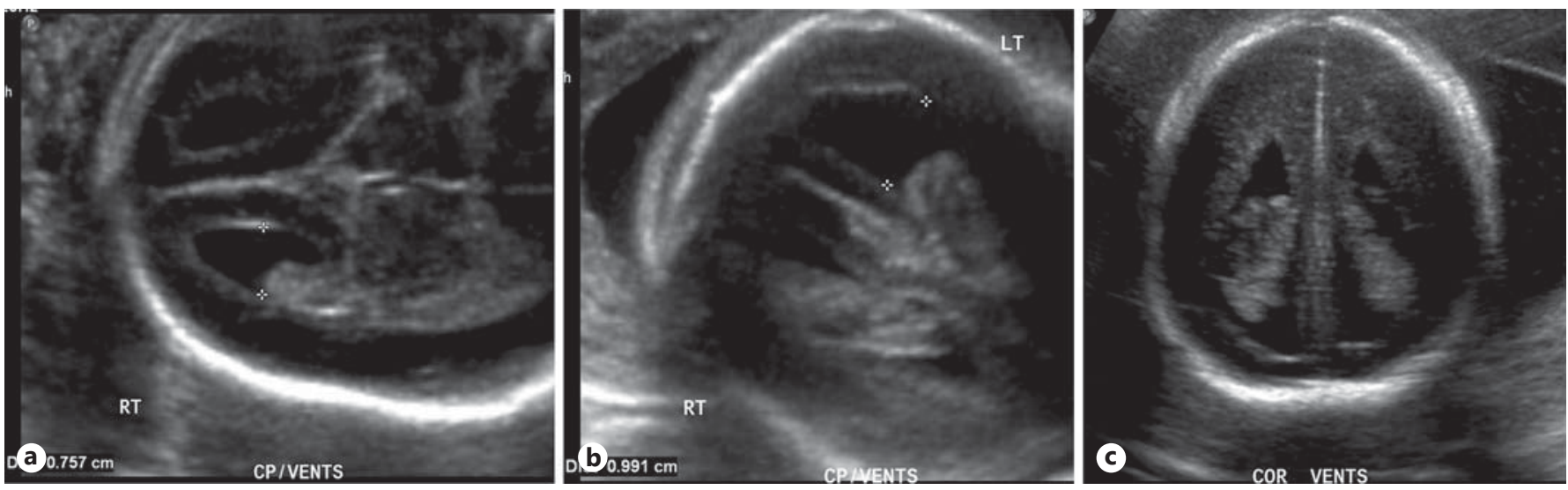

Fig. 21. Asymmetric ventricular size assessed by coronal views at 22 weeks and 6 days. a Axial image of the brain of a fetus with a lumbosacral MMC shows the right lateral cerebral ventricle to be nondilated, measuring $7.6 \mathrm{~mm}$. The left ventricle in the near field

MMC, with up to $1 \%$ of normal fetuses displaying a mild concavity of the frontal bones [17-19, 25]. In addition, it is possible to create the appearance of a lemon sign if the cranium is not imaged in the correct axial plane.

\section{Ventriculomegaly}

Ventriculomegaly (VM) has been reported to occur in $70-90 \%$ of fetuses with SB $[17-19,21,23]$ and is diagnosed when the atria of the lateral cerebral ventricles measure $>10 \mathrm{~mm}$ in diameter on an axial view of the fetal head obtained at the level of the thalami [26] (fig. 20). In fetuses with SB, VM has been noted by ultrasound with increasing prevalence with advancing gestational age [24, $27,28]$. In a study of 53 fetuses with SB, $<50 \%$ of the fetuses diagnosed before 24 weeks of gestational age had VM compared with $90 \%$ that were scanned after 24 weeks [27]. In general, there is a relationship between the severity of VM and both the severity of the posterior fossa malformation and the gestational age of the fetus at the onset of the VM [24]. It is also important to note that the ventricles may be asymmetrically dilated, or one ventricle may be dilated and the other normal in size. For this reason, our protocol includes obtaining measurements of the width of both ventricles, ideally on the axial view. Visualizing the nondependent, near-field ventricle and parenchyma may be challenging due to reverberation artifacts from the fetal skull. To improve our visualization of the cranial anatomy, we add coronal imaging of the fetal head in all cases and TV examination for fetuses in cephalic presentation when necessary (fig. 21). The shape of the lateral ventricular dilation as observed by sonography

Diagnostic Features of Spina Bifida:

Role of Ultrasound is not well seen. b Angled coronal view allows better visualization of the left ventricle, which measures $9.9 \mathrm{~mm}$. c True coronal view of the fetal head demonstrates the ventricular size and anatomy.

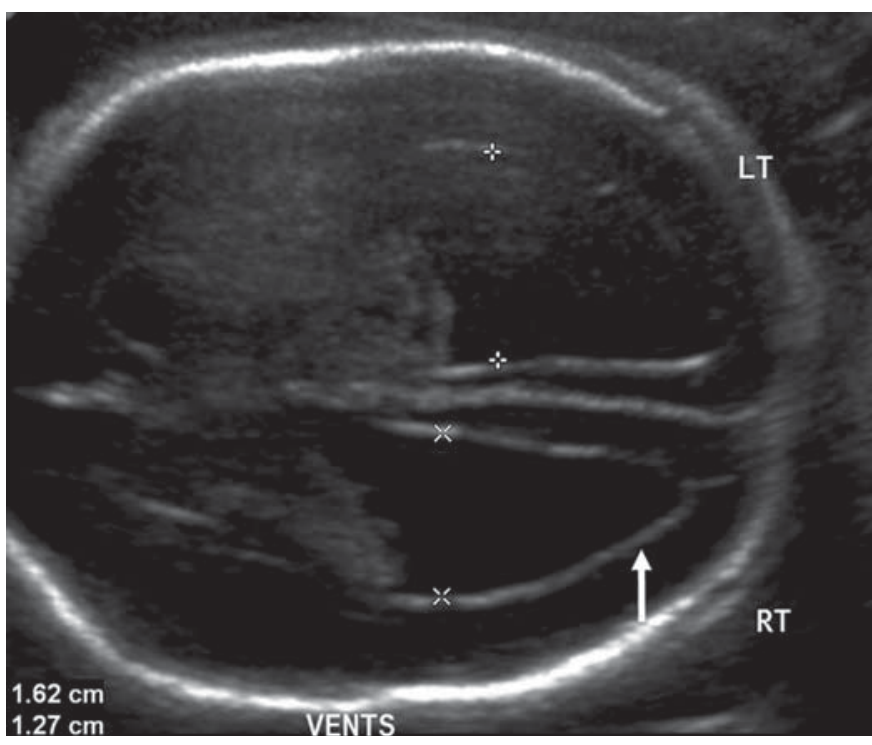

Fig. 22. Asymmetric ventriculomegaly at 22 weeks and 6 days. Axial image of the fetal brain demonstrates asymmetric ventriculomegaly with the right lateral cerebral ventricle dilated to $13 \mathrm{~mm}$ and the left to $16 \mathrm{~mm}$. The tip of the occipital horn of the right ventricle has a pointed shape (arrow), a configuration relatively specific to ventriculomegaly related to MMC as opposed to other neural anomalies.

may also provide an important diagnostic clue for the presence of the Chiari II malformation. Callen and Filly [29] described that the tip of the occipital horn of the lateral ventricles had a pointed shape on the axial view in $70 \%$ of fetuses that proved to have SB and was noted irrespective of coexisting VM (fig. 22). The occipital point- 


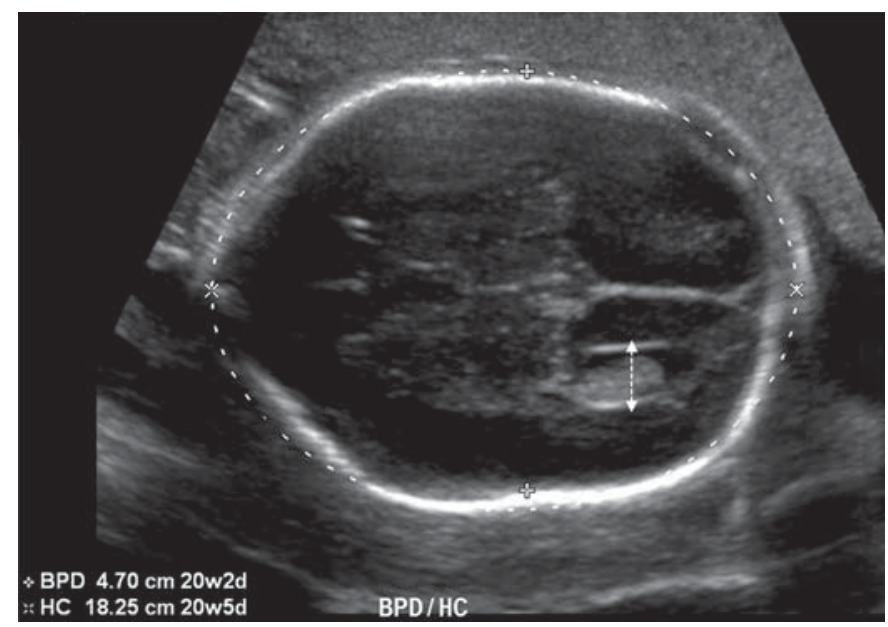

Fig. 23. Microcephaly at 23 weeks. Axial image of the head of a fetus with lumbosacral MMC shows mild frontal bone concavity. The head circumference and the biparietal diameter measurements were less than the 2 nd percentile for the gestational age. The lateral cerebral ventricles were not dilated, with both measuring $5 \mathrm{~mm}$. The ventricle in the far field (dashed arrows) is well seen, but the near-field ventricle is obscured by noise on this axial view.

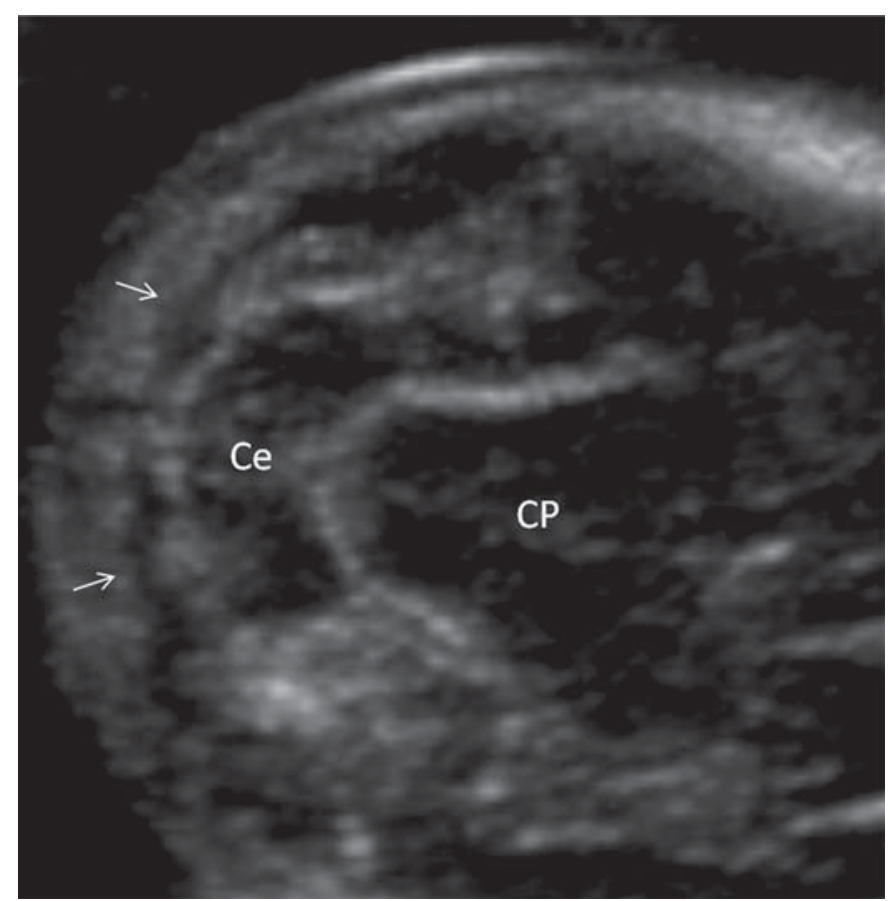

Fig. 24. Hindbrain herniation at 18 weeks and 1 day. $\mathrm{Ce}=$ Cerebellum; $\mathrm{CP}=$ cerebellar peduncles. Angled axial image of the brain of a fetus with a lumbosacral MMC demonstrates a crescentic shape of the compressed cerebellum, called the banana sign, due to the Arnold-Chiari II malformation and a small posterior fossa. Note the near absence of cerebral spinal fluid posterior to the cerebellum (small arrows). ing was more common in fetuses under 24 weeks of gestational age, occurring in $75 \%$ of cases compared with $50 \%$ in gestations between 24 and 35 weeks. Fujisawa et al. [30] also noted that among second-trimester fetuses with VM, a triangular configuration of the occipital horn as observed on coronal TA and TV sonography was helpful in distinguishing VM due to neural tube defects from other central nervous system anomalies. Despite the common presence of VM in SB, microcephaly, defined as a head circumference measurement less than the 10th percentile for gestational age, is also a commonly noted feature of this condition, present in up to $70 \%$ of fetuses [ 17 , 18, 29] (fig. 23).

\section{Hindbrain Herniation Component of the}

\section{Arnold-Chiari II Malformation}

Visualization of hindbrain herniation on ultrasound is the most specific and sensitive finding for the diagnosis of SB and the visualization of a normal-appearing cerebellum, and cisterna magna has a very high negative predictive value in excluding open SB $[23,27,28]$. The sonographic findings of hindbrain herniation on the axial view include the 'banana sign', representing the altered, crescentic shape of the cerebellum in the undersized posterior fossa, a reduction in size or complete effacement of measurable fluid in the cisterna magna, and small size of the cerebellum, which is defined as a transcerebellar diameter less than the 10th percentile for gestational age $[17,18,22,23,30,31]$ (fig. 24). With high-resolution sonography and use of the coronal plane, the fetal cerebellar tonsils can often be visualized below the level of the foramen magnum in affected fetuses (fig. 25).

Quantification of the abnormal shape and small size of the posterior fossa in fetuses with Chiari II malformation has also been described [30]. D'Addario et al. [32] noted that on a midsagittal sonographic view of the fetal cranium, the angle of intersection of a line drawn on the clivus and a line drawn on the posterior occiput, termed the clivus-supraocciput angle, was relatively constant in normal fetuses, measuring $79.3 \pm 6^{\circ}$. The authors found that the angle was constant regardless of gestational age among fetuses undergoing sonography between 16 and 34 weeks' gestational age (fig. 26). The authors compared the clivussupraocciput angle in 44 fetuses with VM due to a variety of central nervous system malformations and found the angle to be significantly reduced $\left(\right.$ mean $=65.1^{\circ}$, range $61-$ 71) for all 12 fetuses with MMC and hindbrain herniation compared with an angle in the normal range or above normal for 30 of 32 fetuses with VM unrelated to MMC [32]. Although most fetuses with MMC will demonstrate 
the classic triad of the banana sign, the lemon sign and $\mathrm{VM}$, posterior fossa abnormalities may be the only cranial finding. Among fetuses with MMC undergoing sonography between 18 and 28 weeks' gestational age, posterior fossa findings were the most commonly noted abnormalities, with a small cerebellum present in $96 \%$ of affected fetuses, a reduced clivus-supraocciput angle $<72^{\circ}$ in $96 \%$ and an effaced cisterna magna in $93 \%$, which compares with a lemon sign in 53\% and VM in $81 \%$ [32].

Although identification of the cranial signs of the Chiari II malformation allows fetal sonography to be diagnostic of virtually all cases of MMC, identification of hindbrain herniation is important for consideration of fetal surgical intervention [5]. Approximately $90 \%$ of fetuses with MMC will have hindbrain herniation detectable by fetal imaging. Hindbrain herniation is assumed to be present by sonography when there is complete effacement of the cisterna magna on axial views. Based on our clinical data, ultrasound overestimates the presence of hindbrain herniation in about $5 \%$ of cases compared with fetal MRI. Directly visualizing the inferior displacement of the cerebellar tonsils on sagittal and coronal views by TA and/or TV scans in the CFDT is considered a more reliable indicator of hindbrain herniation than effacement of the cisterna magna (fig. 6). We routinely perform fetal MRI as part of the evaluation of potential fetal surgical candidates for the assessment of hindbrain herniation and other intracranial abnormalities, and documentation of hindbrain herniation by MRI is an inclusion criterion for fetal MMC surgery candidacy.

\section{Supratentorial Abnormalities in Spinal Dysraphism}

Fetuses with MMC with or without VM may also have a number of supratentorial abnormalities [32-34]. In a study of 25 children with severe MMC, hindbrain herniation and hydrocephalus who died before the age of 2 years, postmortem examination demonstrated disordered cortical migration in $92 \%$, hypoplasia or aplasia of cranial nerve nuclei in $20 \%$, fusion of the thalami in $16 \%$, agenesis of the corpus callosum in $12 \%$ and complete or partial agenesis of the olfactory tract and bulb in $8 \%$ [35]. A retrospective institutional review of children with MMC undergoing MRI found cortical dysplasias and callosal abnormalities in the majority of those examined and gray matter heterotopias in 19\% [33]. Although MRI is considered superior to ultrasound for the diagnosis of cortical dysplasias and heterotopias, several centers with fetal neurosonographic expertise, besides the CFDT, have correctly identified these cortical malformations in utero $[36,37]$. Sonographic findings include overdeveloped

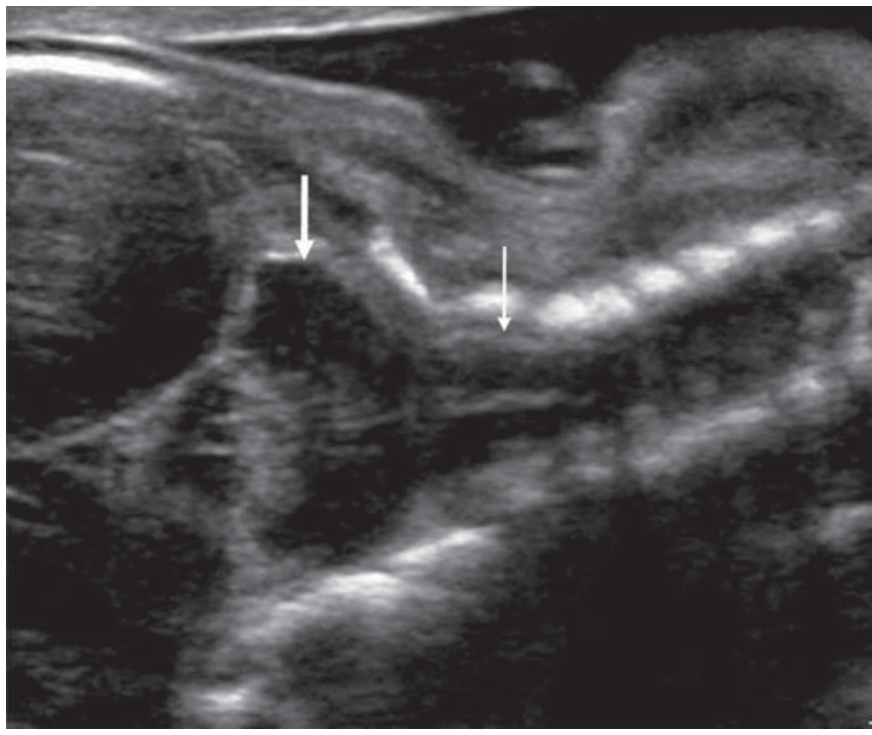

Fig. 25. Severe hindbrain herniation at 19 weeks and 4 days. Coronal image of the posterior fossa and upper cervical region of a fetus with a lumbosacral MMC shows the inferior displacement and compression of the cerebellum (thick arrow) in the small posterior fossa. The cerebellar tonsils (thin arrow) are displaced inferiorly into the upper cervical spine.

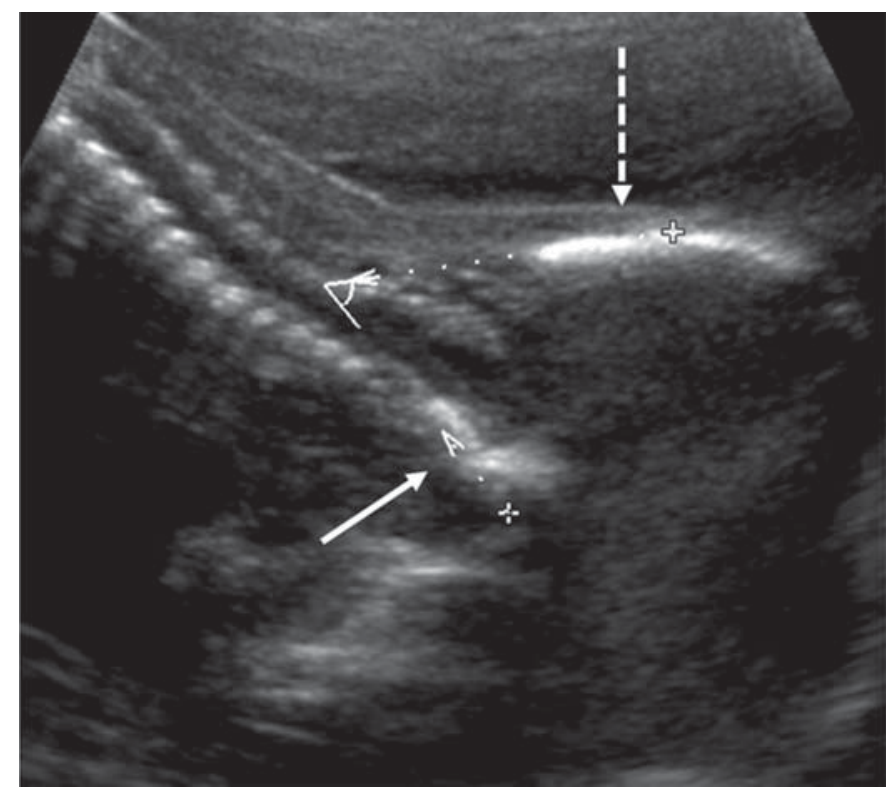

Fig. 26. Abnormal clivus-supraocciput angle at 21 weeks and 4 days. Midline sagittal image of the head of a fetus with a lumbosacral MMC shows that an electronic line drawn on the clivus (closed arrow) and a line drawn on the occiput (dashed arrow) produced an angle of intersection measuring $60^{\circ}$, an angle below the 10 th percentile, indicative of hypoplasia of the cranium overlying the posterior fossa. 
Fig. 27. Gray matter heterotopia at 16 weeks and 6 days. Axial image of the fetal brain of a fetus with a cervicothoracic meningocele as well as a lumbar MMC demonstrates echogenic nodularity of the ventricular lining (small arrows), including a more broad-based echogenic region. Postnatal MRI confirmed cortical heterotopia.
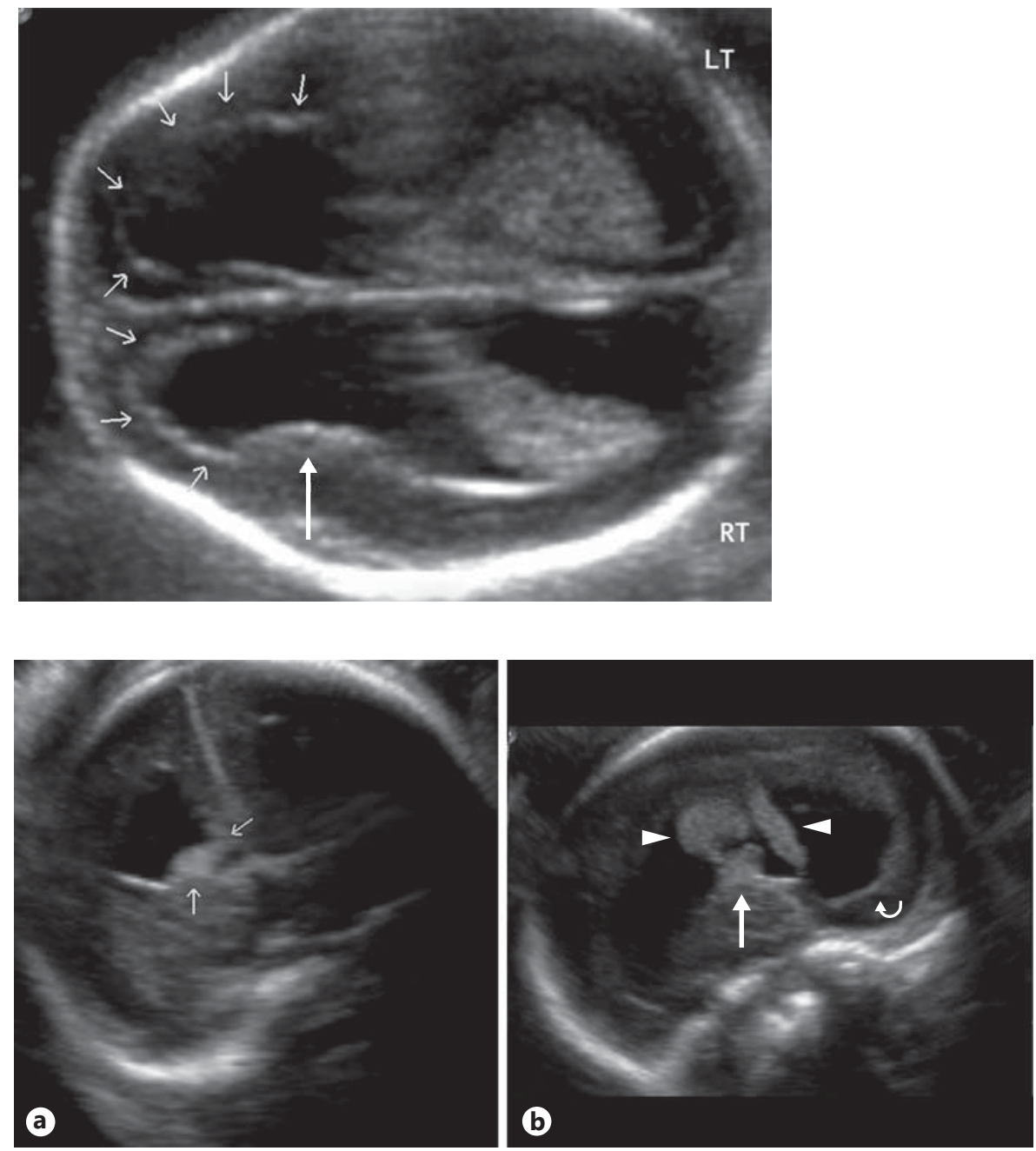

Fig. 28. In utero germinal matrix hemorrhage at 21 weeks and 5 days. a Coronal image of the brain of a fetus with a lumbar myeloschisis shows an echogenic focus (arrows) in the region of the right germinal matrix, indicative of intracranial hemorrhage. b Sagittal view of the right lateral ventricle shows the germinal matrix hemorrhage (arrow), intraventricular thrombus (arrowheads) within the dilated ventricle and irregular thickening of the ependymal lining (curved arrow).

sulci and gyri for gestational age, delayed appearance of landmark sulcation, thin cortex, nodular bulging into the lateral cerebral ventricle, cortical clefts and parenchymal nodules [36] (fig. 27). The diagnosis of these malformations is improved by the use of TV ultrasound when fetuses are in the vertex presentation and expanding the sonographic examination to include sagittal and coronal views of the fetal brain [37]. Additionally, in utero germinal matrix hemorrhage has also been noted in some fetuses undergoing detailed evaluation for MMC repair in our institution. To date, the literature contains few reports of in utero intraventricular hemorrhage, many of which are severe in nature $[38,39]$. In our experience, small germinal matrix hemorrhages are more commonly seen, appearing as echogenic lesions in one or both caudothalamic grooves in association with ependymal thickening as a consequence of clot lysis and retraction [38]. In more advanced cases, there may be a combination of germinal matrix and choroid plexus hemorrhage with layering debris (fig. 28).

\section{Intrinsic Spinal Abnormalities Associated with Spinal Dysraphism}

\section{Syrinx}

Syringomyelia or syrinx, a fluid-filled space within the spinal cord, is sometimes seen in association with MMC (fig. 29). Tsitouras and Sgouros [40] cite an incidence of syrinx in different forms of spinal dysraphism ranging from 21 to $67 \%$. In our experience, a syrinx can be seen sonographically, usually somewhat more easily in the coronal plane, although transverse views can increase the confidence of the diagnosis if a normal cord signature 
Fig. 29. Cervical syrinx with lumbosacral MMC at 21 weeks and 4 days. a Sagittal image of the cervical spine and spinal cord. The syrinx is shown as a fusiform dilatation of the central aspect of the spinal canal extending from approximately $\mathrm{C} 4$ to T4 (arrow). This fetus also had an MMC at the L2-S4 level (not shown). b Transverse image of the cervical spine. The arrows show the border of the dilated central canal. Note that the echogenic 'dot' that normally represents the central canal complex in the spinal cord is absent.
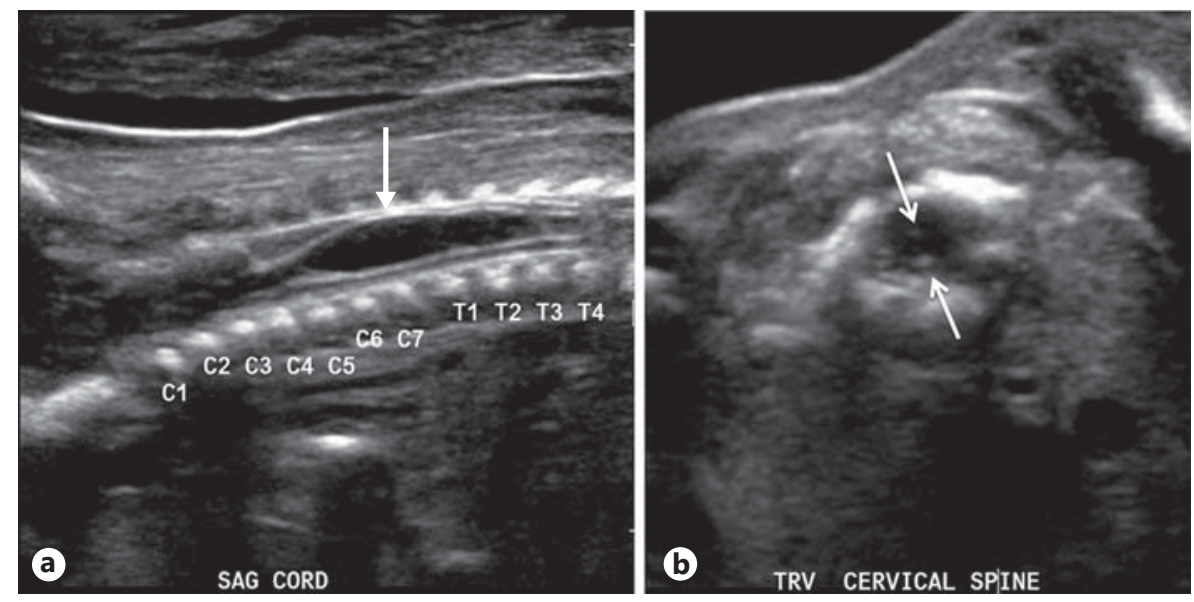

with the central 'dot' representing the central canal complex can be seen proximal and distal to a segment where the central canal is dilated or absent. If the penetration and resolution are sufficient, the expanded outer contour of the cord can be seen as well (fig. 29).

\section{Kyphosis and Scoliosis}

When scoliosis is detected as a complication of MMC, the location of the curvature should be identified. Coronal views of the fetal spine are best for this and one way to describe the location is to note the segment or segments of the spine involved (e.g. 'thoracolumbar') and the approximate level of the center of curvature (e.g. 'centered at L1'). The direction is typically given by the side to which the convex portion of the curve points (e.g. 'convex to the left'; fig. 30). While a description of the curve severity is often qualitative (mild, moderate, severe), it is possible to measure a Cobb angle from coronal images of the fetal spine. For this measurement, it is best if the coronal image is through the ossification centers of the vertebral bodies. Since the vertebral end plates are not ossified in the fetus, the Cobb angle measurement will probably be less accurate than that measured from a spine radiograph.

The principles are the same as for the measurement from a radiograph; the most cephalad and caudal vertebral bodies involved in the scoliosis are identified. Lines parallel to the vertebral bodies (end plates on a radiograph, estimation of the end-plate location or mid-disc space on the ultrasound image) are then extended towards the concave side of the curvature. The angle included between these extended lines is then measured and is the Cobb angle. Because the length of the lines for

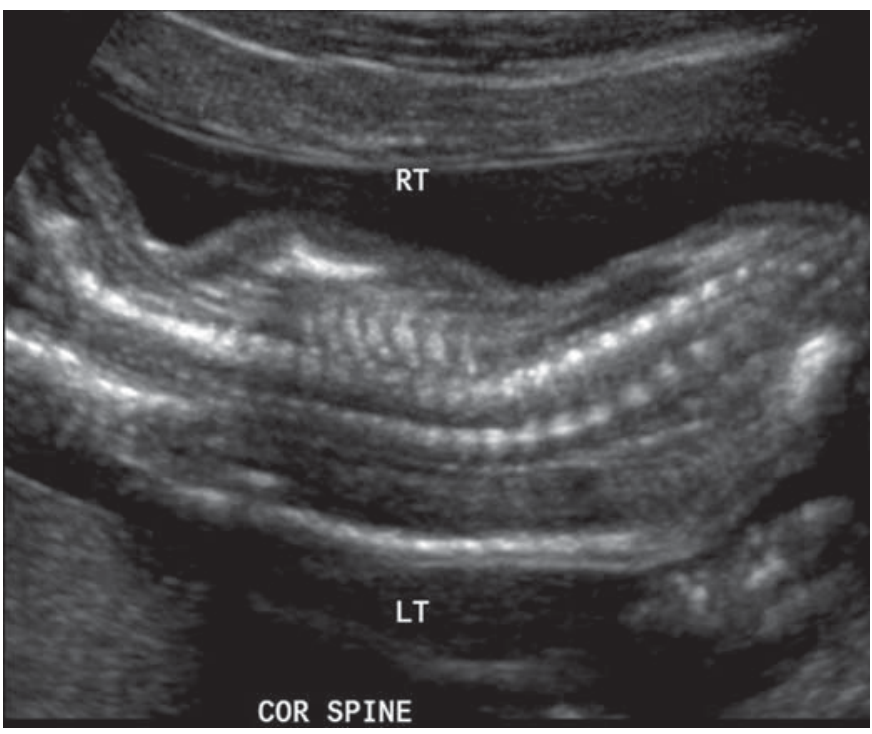

Fig. 30. Scoliosis in association with multiple anomalies at 18 weeks and 5 days. The scoliosis is shown in a coronal image of the spine, which is described as a thoracolumbar scoliosis, convex to the left and centered at L1 (based on the most caudal rib seen as T12). The fetus also had (not shown) a large abdominal wall defect, asymmetric intrauterine growth restriction, and an absent ductus venosus.

slight curvatures will be long before they intersect, an alternate way to measure the Cobb angle is often used. This involves constructing lines perpendicular to each line extended from the vertebral bodies. These two perpendicular lines are then extended until they intersect. This intersection forms four angles: two equal angle pairs. In most instances, the smaller angle is the Cobb angle. Based on 
Fig. 31. Technique for measurement of the Cobb angle. a This is the same fetus as shown in figure 30 . Lines have been drawn in the intervertebral spaces at the vertebral bodies representing the cephalad and caudal extent of the scoliosis. These lines extended will describe an angle (arrowhead) that represents the Cobb angle. In instances where the curve is slight, the lines may extend some distance before intersecting. For this reason, lines perpendicular to those traversing the intervertebral spaces are drawn and extended. The included angle between them (arrow) is (by geometric principles) equal to the Cobb angle as well. b This is the same fetus as shown in figure 30. Lines have been drawn along the centers of the spinal canal and parallel to the lateral elements of the vertebral bodies. The intersection should be at the location of the center of the curvature. The included angle between these lines (arrow) represents the Cobb angle. This is often a measurement method that is easier to use and more accurate than the traditional Cobb angle measurement method used with radiographs (a).

Fig. 32. A 21-week 3-day fetus with multiple congenital anomalies including severe spinal malformation. This 3D image shows a combination of scoliosis (thick arrows) and sharp-angled kyphosis (thin arrow). Note that the ribs are also deformed (arrowheads). Other anomalies (not shown) included a giant omphalocele, a very short umbilical cord, and short humeri and femora. The diagnosis was limb body wall complex.
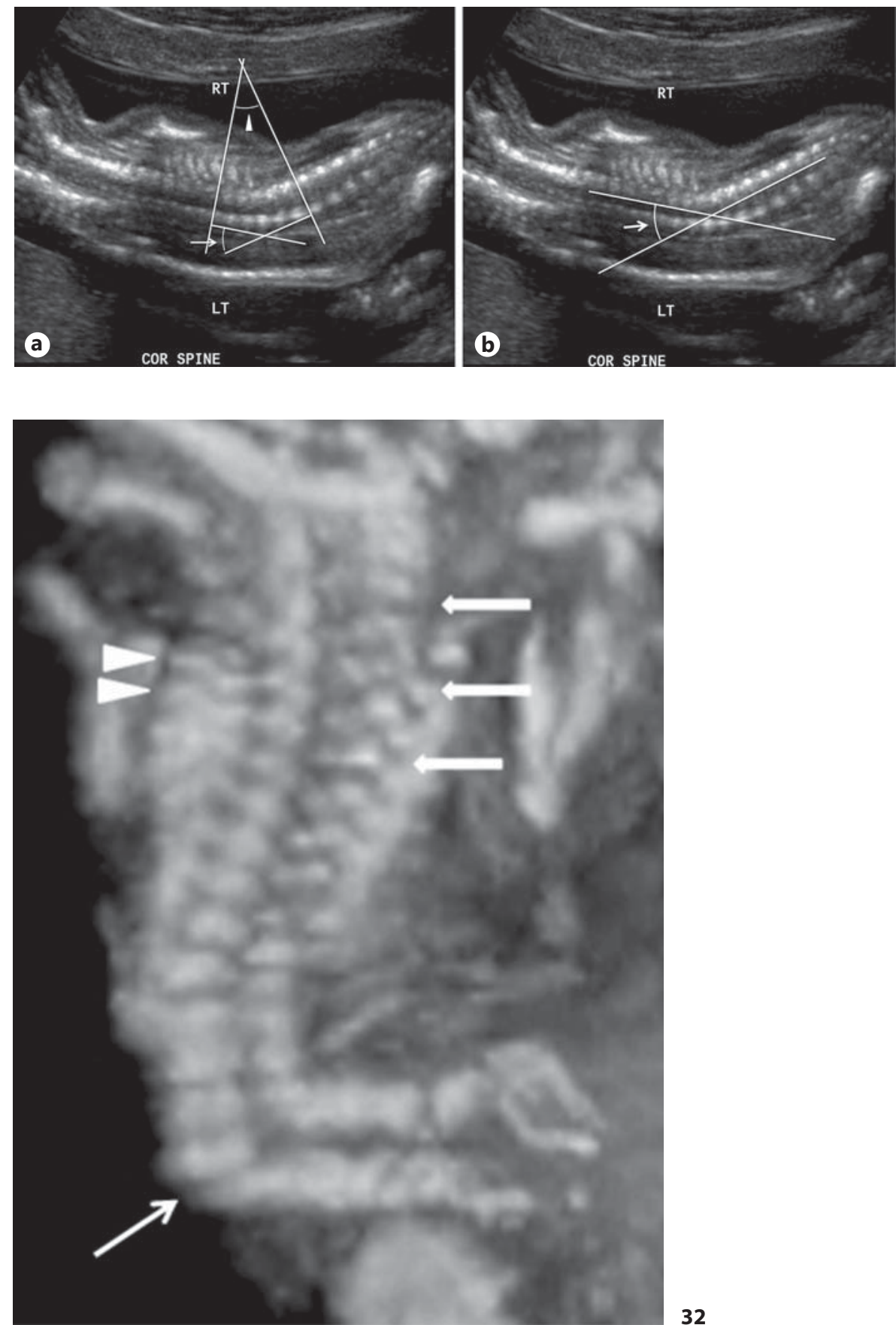

principles of geometry, this is the same angle as that formed by extending the vertebral body lines. For ultrasound, because the vertebral end plates are not fully formed in the fetus, an alternative method for measuring the Cobb angle can be used. This involves drawing straight lines along the segments of the spine cephalad and caudal to the center of the curve. Figure 31 is marked to show how these measurements are made. Those familiar with orthopedics of the spine know that there are a number of other angles measured for scoliosis quantification, but for the usual follow-up imaging of fetuses with scoliosis, the Cobb angle is often sufficient.

Kyphosis, a curvature or angulation of the spine in the sagittal plane, can also be a complication of spina bifida. 

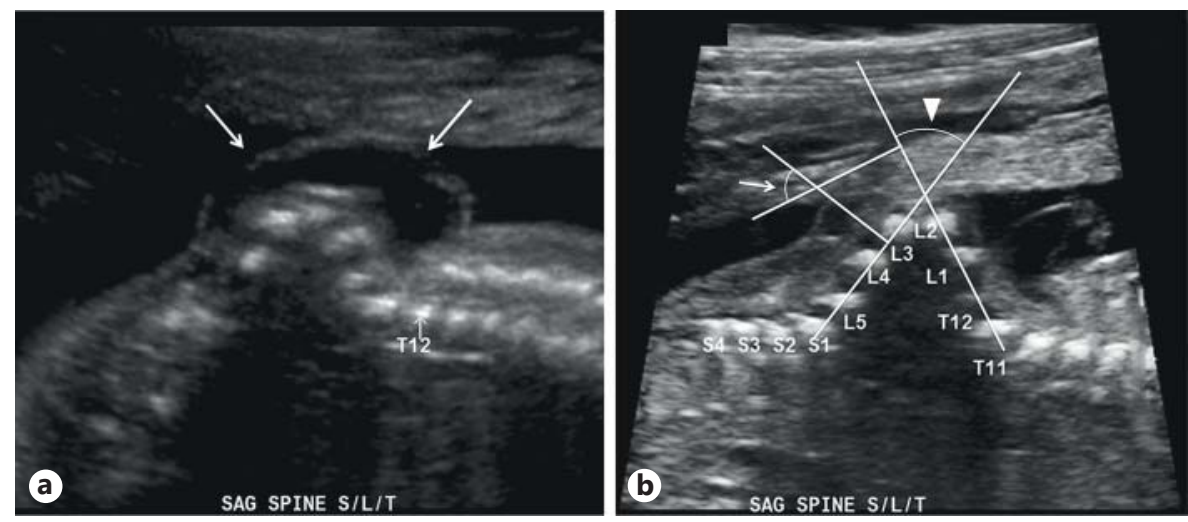

Fig. 33. Thoracolumbar MMC at 23 weeks and 4 days. a A sharpangled kyphosis is present at the level of the MMC sac (arrows). The fetus also had anomalous vertebral bodies, abnormal ribs, cerebral ventriculomegaly, gray matter heterotopia, and bilateral talipes equinovarus. b A sagittal image of the spine centered on the sharp-angled kyphosis. The angle measurement method is to draw lines along the vertebral bodies forming the kyphosis; 2 means of angle measurement are possible. The first is simply to measure the included angle between the 2 drawn lines (arrowhead pointing to the angle arc). The alternative method is to draw perpendiculars to the lines along the vertebral bodies and measure the included angle between them. By geometric principles, this angle (arrow and arc) is the same as that between the lines along the vertebral bodies. This kyphosis would be described as a dorsal, sharp-angled thoracolumbosacral kyphosis extending from T12 to S1 and centered at L2-L3.
The sharp-angled form of kyphosis develops in the MMC fetus as a result of abnormal positioning of the paraspinal muscles. The mechanism of abnormal positioning of the paraspinal muscles as a cause of sharp-angled kyphosis was noted by Cho et al. [41] as a result of a case they reported. Pathological examination of the abortus showed no evidence of abnormal formation of the vertebral bodies or abnormal segmentation. Figure 32 is a $3 \mathrm{D}$ image of a fetal spine that includes both scoliosis and sharp-angled kyphosis.

As with scoliosis, a method of quantitative assessment of kyphosis is important both for follow-up and, in cases of MMC, as a potential exclusion criterion (kyphosis $\geq 30^{\circ}$ ) for fetal surgical MMC therapy [42]. A method similar to the measurement of the Cobb angle is often used. The measurement method is to draw lines along the centers of the most proximal and distal vertebral bodies (usually those just outside the range of vertebra affected by the kyphosis) and measure the acute angle included by these lines. A second method of measurement is analogous to the Cobb angle for scoliosis and involves extending lines from the most cephalad- and caudal-involved vertebral body end plates and measuring the angle between perpendiculars to those lines. Figure 33 depicts a sharp-angled kyphosis and illustrates the angle measurement. When kyphosis is associated with MMC, the location is noted, usually by giving the vertebral body (or bodies) at the center of the curvature (e.g. 'thoracolum- bar kyphosis centered at L1') and the extent (e.g. 'the kyphosis extends from T10 to L4'). The degree of curvature is also measured as described above and the resulting angle is given (e.g. 'the measured kyphosis Cobb angle is $\left.45^{\circ}\right)$.

A close inspection of the spinal canal and cord at the location of the kyphosis is warranted as we have noted cases of diastematomyelia with the bony spur at, or near, the center of the kyphosis curvature and with the split cord evident at this level. The association of kyphosis and diastematomyelia has also been reported by Shorey [43].

\section{Conclusion}

While SB and MMC themselves pose a diagnostic and therapeutic challenge, they can be components of more complex syndromes, associations or complexes. For this reason, a key aspect of evaluation of the fetus with an MMC is to perform a thorough and detailed examination of the entire fetus. Knowledge of what to expect can help increase the detection of associated abnormalities. Though this review has concentrated on the imaging aspects of evaluation, the obstetrical, surgical and genetic aspects are also important and help shape the manner in which imaging is performed. 


\section{References}

$>1$ Budorick NE, Pretorius DH, Grafe MR, Lou $\mathrm{KV}$ : Ossification of the fetal spine. Radiology 1991;181:561-565.

2 Braithwaite JM, Armstrong MA, Economides DL: Assessment of fetal anatomy at 12-13 weeks of gestation by transabdominal and transvaginal sonography. BJ Obstet Gynaecol 1996;103:82-85.

$\checkmark 3$ Zalel Y, Lehavi O, Aizenstein O, Achiron R: Development of the fetal spinal cord: time of ascendance of the normal conus medullaris as detected by sonography. J Ultrasound Med 2006;25:1397-1401.

-4 Aaronson OS, Hernanz-Schulman M, Bruner JP, Reed GW, Tulipan NB: Myelomeningocele: prenatal evaluation - comparison between transabdominal US and MR imaging Radiology 2003;227:839-843.

5 Adzick NS, Thom EA, Spong CY, Brock JW 3rd, Burrows PK, Johnson MP, Howell LJ, Farrell JA, Dabrowiak ME, Sutton LN, Gupta N, Tulipan NB, D’Alton ME, Farmer DL: A randomized trial of prenatal versus postnatal repair of myelomeningocele. $\mathrm{N}$ Engl J Med 2011;364:993-1004.

6 Gregerson DM: Clinical consequences of spina bifida occulta. J Manipulative Physiol Ther 1997;20:546-550.

7 Hertzler DA 2nd, DePowell JJ, Steveson CB, Mangano FT: Tethered cord syndrome: a review of the literature from embryology to adult presentation. Neurosurg Focus 2010; 29:E1.

$>8$ Has R, Yuksel A, Buyukkurt S, Kalelioglu I, Tatli B: Prenatal diagnosis of diastematomyelia: presentation of eight cases and review of the literature. Ultrasound Obstet Gynecol 2007;30:845-849.

$\checkmark 9$ Sarris CE, Tomei KL, Carmel PW, Gandhi CD: Lipomyelomeningocele: pathology, treatment, and outcomes. Neurosurg Focus 2012;33:E3.

-10 Kim SY, McGahan JP, Boggan JE, McGrew $\mathrm{W}$ : Prenatal diagnosis of lipomyelomeningocele. J Ultrasound Med 2000;19:801-805.

$>11$ Gupta DK, Mahapatra AK: Terminal myelocystoceles: a series of 17 cases. J Neurosurg 2005;103(suppl 4):344-352.

-12 Midrio P, Silberstein HJ, Bilaniuk LT, Adzick NS, Sutton LN: Prenatal diagnosis of terminal myelocystocele in the fetal surgery era: case report. Neurosurgery 2002;50:1152-1155.

$\checkmark 13$ Meyer SH, Morris GF, Pretorius DH, James HE: Terminal myelocystocele: important differential diagnosis in the prenatal assessment of spina bifida. J Ultrasound Med 1998;17: 193-197.

14 Rossi A, Piatelli G, Gandolfo C, Pavanello M, Hoffmann C, Van Goethem JW, Cama A, Tortori-Donati P: Spectrum of nonterminal myelocystoceles. Neurosurgery 2006;58:509515
15 Blaas HG, Eik-Nes SH, Isaksen CV: The detection of spina bifida before 10 gestational weeks using two-and three-dimensional ultrasound. Ultrasound Obstet Gynecol 2000; 16:25-29.

16 Sauerbrei EE: The fetal spine; in Rumack CM Wilson SR, Charboneau JW, Levine D (eds): Diagnostic Ultrasound, ed 4. Philadelphia, Elsevier Mosby, 2011, pp 1245-1271.

17 Nicolaides KH, Campbell S, Gabbe SG, Guidetti R: Ultrasound screening for spina bifida: cranial and cerebellar signs. Lancet 1986; $12: 2: 72-74$

18 Campbell J, Gilbert WM, Nicolaides KH, Campbell S: Ultrasound screening for spina bifida: cranial and cerebellar signs in a high risk population. Obstet Gynecol 1987;70: 247-250.

19 Nyberg DA, Mack LA, Hirsch J, Mahony BS: Abnormalities of fetal cranial contour in sonographic detection of spina bifida: evaluation of the 'lemon' sign. Radiology 1988;167: 387-392.

20 McLone DG, Knepper PA: The cause of Chiari II malformation: a unified theory. Pediatr Neurosci 1989; 15:1-12.

21 Cameron M, Moran P: Prenatal screening and diagnosis of neural tube defects. Prenat Diagn 2009;29:402-411.

-22 Van de Hof MC, Nicolaides KH, Campbell J, Campbell S: Evaluation of the lemon and banana in one hundred thirty fetuses with open spina bifida. Am J Obstet Gynecol 1990;162: 322-327.

23 D’Addario V, Rossi AC, Pinto V, Pintucci A, DiCagno L: Comparison of six sonographic signs in the prenatal diagnosis of spina bifida J Perinat Med 2008;36:330-334.

24 Biggio JR Jr, Wenstrom KD, Owen J: Fetal open spina bifida: a natural history of disease progression in utero. Prenat Diagn 2004;24: 287-289.

25 Filly RA: The 'lemon' sign: a clinical perspective. Radiology 1988;167:573-575.

-26 Cardoza JD, Goldstein RB, Filly RA: Exclusion of fetal ventriculomegaly with a single measurement: the width of the lateral ventric ular atrium. Radiology 1988;169:711-714.

27 Babcook CJ, Goldstein RB, Barth RA, Damato NM: Prevalence of ventriculomegaly in association with myelomeningocele: correlation with gestational age and severity of posterior fossa deformity. Radiology 1994;190:703707.

28 Ghi T, Pilu G, Falco P, Segata M, Carletti A, Cocchi G, Santini D, Bonasoni P, Tani G, Rizzo N: Prenatal diagnosis of open and closed spina bifida. Ultrasound Obstet Gynecol 2006;28:899-903.

29 Callen AL, Filly RA: Supratentorial abnormalities in the Chiari II malformation. I. The ventricular 'point'. J Ultrasound Med 2008; 27:33-38.
30 Fujisawa H, Kitawaki J, Iwasa K, Honjo H: New ultrasonographic criteria for the prenatal diagnosis of Chiari type 2 malformations. Acta Obstet Gynecol Scand 2006;85:1426-1429.

-31 Goldstein RB, Podrasky AE, Filly RA, Callen PW: Effacement of the fetal cistern in association with myelomeningocele. Radiology 1989; 172:409-413.

32 D’Addario V, Pinto V, Del Bianco A, Di Naro E, Tartagni M, Miniello G, Serio G: The clivus-supraocciput angle: a useful measurement to evaluate the shape and size of the fetal posterior fossa and to diagnose Chiari II malformation. Ultrasound Obstet Gynecol 2001; 18:146-149.

33 Miller E, Widjaja E, Blaser S, Dennis M, Raybaud C: The old and the new: supratentorial MR findings in Chiari II malformation. Childs Nerv Syst 2008;24:563-575.

34 Hino-Shishikura A, Niwa T, Aida N, Okabe T, Nagaoka T, Shibasaki J: Periventricular nodular heterotopia is related to severity of the hindbrain deformity in Chiari II malformation. Pediatr Radiol 2012;42:1212-1217.

35 Gilbert JN, Jones KL, Rorke LB, Chernoff GF, James HE: Central nervous system anomalies associated with meningomyelocele hydrocephalus, and the Arnold-Chiari malformation; reappraisal of theories regarding the pathogeneses' of posterior neural tube closure defects. Neurosurgery 1986;18:559-564.

36 Malinger G, Kidron D, Schreiber L, Ben-Sira L, Hoffman C, Lev D, Lerman-Sagie T: Prenatal diagnosis of malformations of cortical development by dedicated neurosonography. Ultrasound Obstet Gynecol 2007;29:178-191.

37 Benacerraf BR, Shipp TD, Bromley B, Levine $\mathrm{D}$ : What does magnetic resonance imaging add to the prenatal sonographic diagnosis of ventriculomegaly? J Ultrasound Med 2007; 26:1513-1522.

38 McGahan JP, Haesslein HC, Meyers M, Ford $\mathrm{KB}$ : Sonographic recognition of in utero intraventricular hemorrhage. AJR Am J Roentgenol 1984; 142:171-173.

39 Groothuis AM, Kleine MJ, Oei SG: Intraventricular haemorrhage in utero: a case report and review of the literature. Eur J Obstet Gynecol Reprod Biol 2000;89:207-211.

40 Tsitouras V, Sgouros S: Syringomyelia and tethered cord in children. Childs Nerv Syst 2013;29:1625-1634.

-41 Cho FN, Kan YY, Yang TL, Huang HP, Hsu $\mathrm{PH}, \mathrm{Mai}, \mathrm{MH}$ : Prenatal diagnosis of sharpangled lumbosacral kyphosis with MMC and spina bifida in a fetus. J Ultrasound Med 2004; 22:1115-1119.

42 http://www.chop.edu/export/download/ pdfs/articles/fetal-surg/spina-bifida-referralguide.pdf (last accessed February 7, 2014).

43 Shorey WD: Diastematomyelia associated with dorsal kyphosis producing paraplegia. J Neurosurg 1955;12:300-305. 